\title{
Selective integration of diverse taste inputs within a single taste modality
}

\author{
Julia U. Deere ${ }^{1,3}$, Hannah A. Uttley ${ }^{1}$, Nicole Martinez Santana ${ }^{1}$, and Anita V. Devineni ${ }^{1,2^{*}}$ \\ ${ }^{1}$ Zuckerman Mind Brain Behavior Institute, Columbia University, New York, NY 10027 \\ ${ }^{2}$ Department of Biology, Emory University, Atlanta GA 30322 \\ ${ }^{3}$ Present address: Rockefeller University, New York, NY 10065 \\ *Correspondence: anita.devineni@emory.edu (A.V.D.)
}

\begin{abstract}
Each sensory system contains a diverse repertoire of sensory cells. In the gustatory system, individual taste modalities contain sensory cells that reside in different locations or express different combinations of taste receptors. These different input channels may converge onto common downstream circuits and drive the same behaviors, or they may activate separate pathways to regulate distinct behaviors. To distinguish between these models in Drosophila, we first characterized a repertoire of behaviors induced by optogenetic activation of sugar- or bittersensing neurons. We then activated subsets of bitter-sensing neurons within specific organs. Different subsets elicited a broad and highly overlapping set of behaviors, suggesting that they converge onto common downstream pathways, but we also observed behavioral differences that argue for selective convergence. Transsynaptic tracing experiments provided a neuronal substrate for these results, revealing that bitter neuron subsets connect to overlapping downstream regions with biased connectivity. We characterized one type of second-order bitter neuron that integrates multiple types of bitter input and regulates a subset of bitter-induced behaviors. These results suggest that different bitter inputs are selectively integrated early in the circuit, enabling the pooling of sensory information, and the circuit then diverges into multiple pathways that may have different behavioral roles.
\end{abstract}




\section{INTRODUCTION}

Within a sensory system, sensory neurons are often specialized to detect different types of stimuli. For instance, different mechanoreceptor cells detect different types of touch (Abraira and Ginty, 2013) and different types of cones detect distinct wavelengths of light (Rushton, 1972). Different sensory neurons may also detect stimuli in different locations, such as touch receptors in different parts of the body or photoreceptors in different parts of the retina. A fundamental question thus arises in each sensory system (Estebanez et al., 2018; Rompani et al., 2017; King et al., 2018; Haverkamp et al., 2018; Bates et al., 2020; Jin et al., 2021): when are distinct channels of sensory input merged and when are they kept separate in order to guide behavior? If they are merged, how and where does this integration occur?

Like other sensory systems, the gustatory system contains diverse types of sensory cells. Most attention has been devoted to classifying taste cells based on the taste modality that they detect, such as sweet or bitter (Liman et al., 2014). Although neurons for each taste modality are often studied as a homogeneous population, each modality contains a repertoire of functionally diverse sensory cells. This diversity has been well-characterized in Drosophila melanogaster, which contains sweet- or bitter-sensing neurons residing in multiple organs and expressing distinct combinations of sweet or bitter taste receptors (Scott, 2018; Weiss et al., 2011; Ling et al., 2014; Fujii et al., 2015; Chen and Dahanukar, 2017).

Flies harbor taste sensory neurons in external organs such as the legs, wings, and labellum (the distal segment of the proboscis) as well as internal organs such as the pharynx (Scott, 2018). Because different taste organs experience taste stimulation under different conditions, taste neurons in different organs are thought to have distinct behavioral roles (Dethier, 1976; Scott, 2018). This model has been most clearly elucidated for appetitive tastes such as sugar, which elicits a sequence of behaviors regulated by specific organs (Thoma et al., 2017; Scott, 2018; Mahishi and Huetteroth, 2019). Flies first detect the presence of sugar with the legs. Depending on the sugar concentration and the fly's hunger state, the fly may then extend its proboscis to contact the food with the labellum. Labellar taste stimulation may control the initiation of food intake, which leads to stimulation of pharyngeal taste neurons that regulate the duration of food ingestion (LeDue et al., 2015; Yapici et al., 2016; Joseph et al., 2017).

Sugar- or bitter-sensing neurons within a single organ also exhibit functional diversity. For example, four classes of bitter-sensing neurons (S-a, S-b, I-a, and I-b) have been identified in the labellum based on the combination of gustatory receptors ( $\mathrm{Grs}$ ) that they express, and they show different response profiles when stimulated with bitter tastants (Weiss et al., 2011). Multiple classes of bitter neurons in the leg and pharynx have also been identified (Ling et al., 2014; Chen and Dahanukar, 2017).

Thus, an individual taste modality such as "bitter" does not represent a single pathway, but instead comprises multiple channels of sensory input within and across organs. How are these diverse channels of bitter input translated into behavioral output? One possibility is that all channels of bitter input are integrated and regulate a common set of behaviors, representing an 
"integrative model". Alternatively, different channels of bitter input may be processed separately to regulate distinct behaviors, a "segregated processing model". This would allow the fly to execute specific aversive responses depending on the compound that is detected or the organ that detects it. In support of a segregated processing model, several studies have shown that different subsets of sugar- or bitter-sensing neurons have different behavioral roles (Joseph and Heberlein, 2012; Thoma et al., 2016; Murata et al., 2017; Chen et al., 2019; Chen et al., 2021; Chen et al., 2022), with most of these studies comparing sugar-sensing neurons in different organs. Moreover, taste neurons in different organs project axons to spatially segregated regions within the subesophageal zone (SEZ), the primary taste area of the brain, suggesting that they may activate distinct downstream pathways (Wang et al., 2004; Kwon et al., 2014). Recent findings demonstrating that bitter-sensing neurons in different organs show different response dynamics also suggest that they may have distinct functional roles (Devineni et al., 2021).

In this study we aimed to systematically test whether inputs from different subsets of neurons within a single taste modality are integrated or processed separately to regulate behavior. We first characterized a broad set of behaviors that are robustly induced by optogenetic activation of sugar- or bitter-sensing neurons. We then activated subsets of bitter neurons in specific organs. Most bitter neuron subsets elicited a broad and overlapping set of behaviors, suggesting that these inputs converge onto common downstream pathways, but we also observed differences that argue for selective convergence. Transsynaptic tracing experiments revealed a neuronal substrate for these behavioral results, showing that different bitter neuron subsets connect to overlapping second-order taste regions with biased connectivity. We identified one type of second-order bitter neuron that integrates bitter input from multiple organs and regulates a subset of bitter-induced behaviors. Together, these results suggest that bitter inputs from different organs are selectively integrated early in the taste circuit and drive largely overlapping behaviors, contrasting with the conventional model that different organs have different functions. The circuit then diverges into multiple pathways that may have different behavioral roles.

\section{RESULTS}

\section{Sugar- and bitter-sensing neurons drive opposing changes in locomotion}

Most studies of taste have focused on feeding-related behaviors. We wished to characterize a broader set of behaviors induced by appetitive or aversive tastes. We chose to optogenetically activate sugar- or bitter-sensing neurons rather than using natural tastes so that we could precisely control the timing and duration of taste stimulation and target genetically defined neuronal subsets. We used the light-activated channel Chrimson to drive neuronal activation (Klapoetke et al., 2014). We targeted its expression to sugar- or bitter-sensing neurons using Gal4 drivers that are expressed in nearly all known sugar-sensing (Gr64f-Gal4) or bitter-sensing (Gr33a-Gal4) neurons throughout the body (Ling et al., 2014; Weiss et al., 2011; Chen and Dahanukar, 2017). Chrimson has been previously used to activate taste sensory neurons (Klapoetke et al., 2014; Musso et al., 2019; Moreira et al., 2019), and in an earlier study we 
used calcium imaging to show that Chrimson-expressing taste sensory neurons are effectively activated by red light (Devineni et al., 2021).

We first tested how taste neuron activation affects locomotion. Previous studies have reported taste-induced locomotor changes that are related to foraging and feeding. For instance, the taste of sugar suppresses locomotion, which enables a fly to initiate feeding (Flood et al., 2013; Thoma et al., 2016). We tested the effect of taste neuron activation on locomotion by tracking flies' movement in a circular arena (Figure 1A-B) (Aso et al., 2014). We delivered 5 sec light stimulation at three different intensities ("low", "medium", and "high"), which corresponded to 4, 20 , and $35 \mu \mathrm{W} / \mathrm{mm}^{2}$. Activating sugar-sensing neurons strongly suppressed locomotion at all light intensities (Figure 1C), consistent with previous studies using natural sugar (Flood et al., 2013; Thoma et al., 2016). Both forward velocity and turning were suppressed at light onset (Figure 1C), and the fraction of flies moving decreased to nearly zero (Figure 1 - Figure Supplement 1A). All of these locomotor changes were transient, as they recovered within 2-3 seconds even though the light stimulation was still on. The transient nature of these effects may be because flies quickly determine that there is no food source actually present and resume normal behavior.

In contrast to sugar neuron activation, activating bitter neurons strongly increased the average forward velocity (Figure 1D) as well as the fraction of flies moving (Figure 1 - Figure Supplement 1B). This effect was strongest at light onset and gradually diminished over 5 sec. When light stimulation was turned off, flies immediately stopped moving (Figure 1D and Figure 1 - Figure Supplement 1B-C). This post-light locomotor suppression persisted for nearly a minute (Figure 1 - Figure Supplement 1D). Light onset also elicited a transient increase in turning that lasted $\sim 1 \mathrm{sec}$ or less (Figure 1D). These results suggest that when a fly senses a bitter stimulus, it shows a sequence of stereotyped behaviors: 1) it first turns in an attempt to orient away from the stimulus, 2) it increases its forward velocity in an attempt to run away from the stimulus, and 3) when the stimulus disappears, it freezes in place because it may perceive its current position to be a safe (bitter-free) location.

To determine whether the locomotor effects of sugar or bitter neuron activation are modulated by hunger state, we repeated these experiments in starved flies. Starvation has been shown to enhance feeding responses to sugar, whereas it suppresses the aversive effect of bitter (Inagaki et al., 2012; Marella et al., 2012; Inagaki et al., 2014; LeDue et al., 2016). The ability of sugar neuron activation to suppress locomotion was strongly enhanced in flies starved for one day (Figure 1E). In contrast, the locomotor effects of bitter neuron activation did not differ between fed flies and flies starved for either one or two days (Figure 1F). Thus, the locomotor effects of activating sugar neurons, but not bitter neurons, depend on hunger state.

Given that sugar and bitter neuron activation elicited opposing effects on locomotion, we tested the effect of activating them simultaneously. Activating both sugar and bitter neurons elicited effects similar to activating bitter neurons alone: forward velocity and turning were strongly increased (Figure 1G-H), indicating that the locomotor enhancement driven by bitter neurons dominates over the locomotor suppression driven by sugar neurons. However, closer 
examination of the light onset period revealed that activating both types of neurons caused very brief locomotor suppression, resulting in a longer delay to reach peak velocity and turning as compared to activation of bitter neurons alone (Figure $1 \mathrm{G}-\mathrm{H}$ ). This trend was not significant for peak velocity (Figure 1G; $p=0.069$ for starved flies), but was robust and highly significant for peak turning (Figure $1 \mathrm{H}$ ). The delay to peak turning was also enhanced in starved flies compared to fed flies (Figure $1 \mathrm{H}$ ). These results suggest that when sugar and bitter neurons are co-activated, the two inputs are integrated differently across time: sugar input drives behavior at very early time points after light onset, but bitter input dominates throughout the rest of the stimulation period. Under natural conditions, these effects may lead to sequential behaviors when flies encounter a mixture of sugar and bitter: the taste of sugar causes flies to briefly stop and consider feeding, but sufficiently strong bitter taste overrides this program and causes them to run away.

\section{Sugar- and bitter-sensing neurons have opposing effects on positional preference}

We next tested the effect of sugar or bitter neuron activation on positional preference: whether flies prefer or avoid residing in areas where those taste neurons are activated. Previous studies have shown that flies display positional preference for sugar and avoidance of bitter (Marella et al., 2006; Joseph and Heberlein, 2012; Thoma et al., 2016). Positional preference thus correlates with the effects of these taste modalities on feeding, but it is a distinct behavior that may be displayed in the absence of feeding and is mediated by different motor circuits (i.e., motor neurons controlling the legs versus the feeding organ).

To assess the effect of taste neuron activation on positional preference, we presented light in two opposing quadrants of the arena and quantified the flies' preference for residing in the light quadrants (Figure 2A). Activating sugar neurons at low light intensity elicited strong positional preference in one-day starved flies, but only a weak, non-significant effect in fed flies (Figure 2B-D). Unexpectedly, this attraction diminished at medium light intensity and shifted to aversion at high intensity (Figure 2C-D and Figure 2 - Figure Supplement 1A-B). This high intensity aversion has been previously observed (Mohammad et al., 2017) and may reflect a compensatory change in the sugar-sensing neurons or downstream circuits (see Discussion). Although locomotor suppression can lead to positional preference by making flies more likely to remain in a certain area, our results suggest that these two behaviors are clearly dissociable. Locomotor suppression was observed at all light intensities in both fed and starved flies (Figure $1 \mathrm{C}$ and $1 \mathrm{E})$, whereas positional preference was only observed in starved flies at the lowest intensity (Figure 2B-D).

Activating bitter-sensing neurons elicited strong positional aversion at all light intensities (Figure $2 \mathrm{E}-\mathrm{G}$ and Figure 2 - Figure Supplement 1C-D). The degree of aversion was similar in fed flies and flies starved for one or two days (Figure 2E-G). This aversion may be at least partially mediated by the locomotor effects of bitter activation (Figure 1). Increased locomotion and turning at the onset of bitter activation would increase the likelihood that flies exit the light quadrants, and locomotor suppression at light offset would make flies more likely to stay in the non-light quadrants. However, we observed some flies crossing back and forth between the light 
and non-light quadrants before settling in the non-light quadrants, suggesting that a more active choice process may also be occurring.

We then tested the effect of activating sugar and bitter neurons simultaneously at low light intensity in one-day starved flies, conditions under which sugar neurons elicit strong attraction and bitter neurons elicit strong aversion. Activating both sugar and bitter neurons elicited a similar degree of avoidance as activating bitter neurons alone (Figure $2 \mathrm{H}-\mathrm{J}$ ), demonstrating that the aversion elicited by bitter neurons completely dominates over the attraction elicited by sugar neurons. Fed flies also showed similar behavior (Figure 2J).

\section{Sugar- and bitter-sensing neurons drive opposing types of learning}

In addition to their effects on innate behavior, sugar and bitter stimuli have been used as reinforcement cues for olfactory learning (Das et al., 2014; Schwaerzel et al., 2003; Huetteroth et al., 2015). We therefore asked whether activation of sugar- or bitter-sensing neurons could drive learned preference or aversion toward an odor. We followed previously established protocols for testing short-term learning and memory (Aso and Rubin, 2016). Specifically, we presented one odor (the conditioned stimulus, CS+) while activating taste neurons with light, then presented a different odor (the CS-) without neuronal activation, and finally presented both the CS+ and CS- odors in opposing quadrants and assessed where the flies preferred to reside (Figure 3A). Pairing odor with sugar neuron activation elicited strong learned preference for the $\mathrm{CS}+$ in starved flies, but not fed flies (Figure 3B-D). Pairing odor with bitter neuron activation elicited learned aversion to the CS+ in both fed and starved flies, and starved flies showed a stronger response (Figure 3E-G). Simultaneously activating sugar and bitter neurons during learning in starved flies resulted in CS+ aversion, similar to activating bitter neurons alone (Figure $3 \mathrm{H}-\mathrm{I}$ ).

Together, these data show that optogenetic activation of sugar- or bitter-sensing neurons drives opposing effects on locomotion, positional preference, and learning (Figure 4A). In all three behavioral paradigms the effect of sugar neurons, but not bitter neurons, was strongly modulated by hunger state (Figure 4A). In addition, in all paradigms the simultaneous activation of both neuronal classes revealed that the behavioral effects of the bitter pathway generally dominate over the sugar pathway.

\section{Activating subsets of bitter neurons affects locomotion, preference, learning, and feeding responses}

Having characterized a set of behaviors that are driven by sugar or bitter neuron activation, we next asked how individual channels of taste input within a single modality contribute to those behaviors. We focused on bitter taste for these studies. Bitter input from different organs or neuronal classes could be integrated to regulate the same behaviors (Figure 4B, left) or processed separately to regulate distinct behaviors (Figure 4B, right). We aimed to distinguish between these models by activating individual subtypes of bitter neurons within specific organs. We used previously characterized Gal4 lines (Weiss et al., 2011; Ling et al., 2014; Kwon et al., 
2014; Chen and Dahanukar, 2017) to target four subsets of bitter neurons: 1) bitter neurons in the leg ( $\mathrm{Gr} 58 \mathrm{c}-\mathrm{Gal}$, labeling the major class of tarsal bitter cells comprising 7 of the 8 tarsal bitter cells per side); 2) bitter neurons in the labellum belonging to classes S-a and I-a (Gr59cGal4, labeling 13 of the 19 labellar bitter neurons per hemisphere); 3) bitter neurons in the labellum belonging to class S-b (Gr22f-Gal4, labeling 3 of the 19 labellar bitter neurons per hemisphere); and 4) a pharyngeal bitter neuron termed V6 (Gr9a-Gal4, labeling 1 of the $\sim 5$ putative pharyngeal bitter neurons). Because these Gal4 lines label different numbers of neurons and may also vary in their expression strength, we focused on analyzing whether the activation of each neuronal subset could drive any significant change in each behavior rather than comparing the strength of behavioral effects across lines.

We first tested the effect of each bitter neuron subset on locomotion. Activating any of the four subsets enhanced locomotion for at least one of the three light intensities (Figure 5A and Figure 5 - Figure Supplement 1A). Three of the four subsets (all except labellar S-b neurons) also elicited an increase in turning at stimulation onset and locomotor suppression after stimulation was turned off (Figure 5B and Figure 5 - Figure Supplement 1), although the latter effect was only significant for two lines. These results show that bitter neuron subsets in three different organs - the leg, labellum, or pharynx - can drive aversive locomotor behaviors.

Activating three of the four bitter neuron subsets elicited innate positional aversion (Figure 5C and Figure 5 - Figure Supplement 2), and two of the four subsets elicited learned odor aversion (Figure 5D). Labellar S-b neurons were the only subset that failed to elicit significant effects in either paradigm. Pharyngeal neurons did not elicit a significant learned response despite driving strong innate aversion.

As an additional readout of bitter aversion, we tested the ability of bitter neurons to suppress the proboscis extension response (PER). PER is a well-established appetitive response that represents initiation of feeding and is inhibited by bitter compounds (Dethier, 1976; Scott, 2018; Wang et al., 2004). Activating all bitter-sensing neurons strongly suppressed PER to sugar, as did activation of any of the four bitter neuron subsets (Figure 5E).

Together, these experiments show that different subsets of bitter neurons drive broad and largely overlapping behavioral responses (Figure 6). The fact that all neuronal subsets had a clear effect on at least one aversive behavior suggests that each set of neurons was effectively activated by Chrimson. Labellar S-b neurons had the sparsest effect and only affected two of the six behavioral measures analyzed (Figure 6), but we also noted that the corresponding Gal4 line seemed to have weaker expression than the other lines (data not shown). The other three bitter neuron subsets, residing in three different organs, were all capable of driving aversive behaviors relating to locomotion, preference, and feeding (PER). Two of the three subsets (leg and labellar S-a + I-a neurons) also elicited aversive learning (Figure 6).

Overall, these results support an integrative model of taste processing (Figure 4B, left) in which bitter inputs from different organs converge onto downstream pathways to drive common aversive behaviors. However, the fact that not all neuronal subsets could elicit all behaviors, or 
preferentially elicited some behaviors over others, suggests that these inputs may be selectively integrated: downstream pathways driving behavior may receive stronger input from some neuronal subsets than others.

\section{Bitter input from different organs is relayed to overlapping downstream regions}

The behavioral results described above suggest that taste circuits in the brain integrate bitter input from different organs. Where does this integration occur? Cross-organ integration could occur early in the taste circuit, potentially at the first synapse, or could occur much later in the circuit. To test whether integration may occur at the first synapse, we used the transsynaptic tracing technique trans-Tango (Talay et al., 2017) to identify neurons that are postsynaptic to bitter sensory cells, termed second-order bitter neurons. We first labeled the entire population of second-order bitter neurons by tracing neurons postsynaptic to Gr33a-Gal4-expressing cells. We observed many local neurons projecting within the SEZ as well as three major projections to distinct regions of the superior protocerebrum: a lateral, medial, and mediolateral tract (Figure 7A). This generally resembles the pattern of second-order sugar neurons labeled with transTango (Talay et al., 2017) as well as second-order bitter neurons labeled in recent studies (Chen et al., 2019; Snell et al., 2020). These experiments reveal that at the first synapse, the bitter taste circuit diverges into at least three downstream pathways projecting out of the SEZ.

We next used trans-Tango to label neurons postsynaptic to specific subsets of bitter sensory cells, using the same Gal4 lines used to test behavior. Tracing neurons postsynaptic to bitter neurons in the leg labeled all three projection tracts, although the medial tract was labeled relatively weakly (Figure 7B). Tracing from S-a + I-a neurons in the labellum also labeled all three tracts (Figure 7B). The medial tract was labeled more strongly than when tracing from the leg, whereas the lateral tract was labeled more weakly. Finally, tracing neurons postsynaptic to the pharyngeal bitter neurons labeled the medial tract almost exclusively, although the mediolateral tract occasionally showed weak labeling (Figure 7B). A recent study tracing postsynaptic partners of pharyngeal taste neurons reported similar results, although the mediolateral tract was more clearly labeled in that study (Chen et al., 2019). Together, these results show that: 1) bitter neurons in each organ synapse onto multiple second-order pathways, and 2) each projection tract receives input from multiple organs, although it is not clear whether different inputs converge onto the same individual second-order neurons. Interestingly, bitter inputs show biased connectivity to second-order taste regions, with bitter neurons in different organs preferentially connecting to different projection tracts. This selective integration could allow downstream taste pathways to be activated more strongly by some organs than others.

\section{Second-order mISEZt neurons receive bitter input from the leg and labellum}

In order to study specific types of second-order bitter neurons, we searched for driver lines that label them. Our trans-Tango experiments generated dense staining patterns that made it difficult to discern the morphology of individual cells (Figure 7). However, second-order bitter neurons appear to be anatomically similar to second-order sugar neurons (Talay et al., 2017; Chen et al., 
2019), and in fact some of them may be the same neurons receiving both types of input (Snell et al., 2020). The morphology of individual second-order sugar neurons has been previously described using stochastic labeling (Talay et al., 2017). We therefore proceeded on the assumption that second-order bitter neurons may have a similar morphology as second-order sugar neurons, and we searched the Janelia FlyLight expression database (Jenett et al., 2012) for lines labeling them.

This approach identified multiple Gal4 lines that appeared to label second-order taste neurons comprising the mediolateral tract. We refer to them as mISEZt neurons based on the terminology in Talay et al. (2017). As visible in the first line we identified, R29F12-Gal4, mISEZt neurons have cell bodies just dorsal to the antennal lobes, a V-shaped projection in the SEZ, and a circular projection in the superior lateral protocerebrum (SLP) (Figure 8A, top). Colabeling bitter-sensing neurons and mISEZt neurons revealed that bitter sensory axons overlap with the medial branch of the mISEZt projection in the SEZ (Figure 8A, bottom).

To confirm that these mISEZt neurons receive bitter input, we monitored their responses to taste stimulation using calcium imaging. The projections of mISEZt neurons in the SEZ and SLP could be clearly identified based on their location and morphology. mISEZt projections in both the SEZ and SLP responded to bitter stimulation of the labellum or leg (Figure 8B-C), confirming that these neurons receive bitter input from multiple organs. Water or sugar applied to the labellum elicited a much weaker response in the SEZ ( $50 \%$ of the bitter response; Figure $8 \mathrm{~B})$ and no response in the SLP (Figure $8 \mathrm{C}$ ). The sugar response was not higher than the water response. Water or sugar responses were not observed in either the SEZ or SLP with tarsal stimulation (Figure 8B-C). The addition of sugar also did not modulate the response to labellar bitter stimulation (Figure 8 - Figure Supplement 1A), suggesting that bitter-responsive mISEZt neurons do not receive inhibition from the sugar pathway. Thus, the mISEZt neurons labeled with R29F12-Gal4 appear to include second-order bitter neurons, but not second-order sugar neurons.

mISEZt neurons showed different response dynamics when bitter was applied to the labellum versus the leg, as previously reported for bitter sensory neurons in these organs (Devineni et al., 2021). Bitter stimulation of the labellum elicited transient responses at bitter onset (ON response) and offset (OFF response), whereas bitter stimulation of the leg elicited a more sustained ON response with no OFF response (Figure 8B-C). To directly compare the magnitude of mISEZt responses to labellar and tarsal bitter stimulation, we imaged responses in the same flies (Figure 8 - Figure Supplement 1B). Labellar stimulation elicited stronger activity at bitter onset than tarsal stimulation ( $70 \%$ versus $\sim 30 \% \Delta F / F)$, suggesting that $\mathrm{mISEZt}$ neurons are more strongly driven by labellar inputs than leg inputs.

Tarsal and labellar bitter stimulation also produced different spatial patterns of activation in the SEZ (Figure 8 - Figure Supplement 1C). Responses to tarsal stimulation were localized to a small region located at the dorsolateral edge of the larger region activated by labellar stimulation. This is consistent with the known projection patterns of tarsal and labellar bitter sensory neurons (Wang et al., 2004; Kwon et al., 2014) and our colabeling experiment (Figure 
8A, bottom). Thus, bitter inputs from different organs likely activate different dendrites on mISEZt neurons.

In the SLP, the spatial pattern of mISEZt activation was similar when bitter was applied to either organ, although the level of activation was stronger with labellar stimulation (Figure 8 - Figure Supplement 1D). We observed individual puncta, likely representing axon terminals of single mISEZt cells, that were activated by bitter stimulation of either organ (Figure 8 - Figure Supplement 1D). These results suggest that individual mISEZt cells receive convergent input from the labellum and leg. mISEZt cell bodies, which are located far from either the SEZ or SLP projections (Figure 8A), did not show bitter responses (data not shown), preventing us from unequivocally imaging responses in single mISEZt cells.

Finally, we tested whether labellar bitter responses in mISEZt neurons depend on the identity of the bitter compound and the recent history of bitter stimulation, as shown for labellar bitter sensory neurons (Devineni et al., 2021). Indeed, mISEZt neurons responded to different bitter compounds with different response dynamics. Relative to the strength of the ON response, denatonium and quinine elicited strong OFF responses, caffeine elicited a slightly weaker OFF response, and L-canavanine elicited no OFF response at all (Figure 8 - Figure Supplement $1 \mathrm{E})$. These compound-dependent dynamics resemble the responses of sensory neurons (Devineni et al., 2021). mISEZt responses also showed an experience-dependent effect resembling that of labellar bitter-sensing neurons: with repeated bitter stimulation, the bitter ON response habituated much more strongly than the OFF response (Figure 8 - Figure Supplement $1 \mathrm{~F})$.

Together, these results show that the mISEZt neurons labeled by R29F12-Gal4 are secondorder bitter neurons. The taste selectivity and response dynamics of mISEZt bitter neurons closely resemble those of bitter sensory neurons. The major transformation we observe is that mISEZt neurons integrate bitter input from at least two different organs, the leg and labellum.

\section{Second-order mISEZt bitter neurons regulate a subset of behaviors}

We next tested the role of mISEZt bitter neurons in regulating behavior. Because R29F12-Gal4 labels a number of neurons other than mISEZt cells (Figure 8A), we used a split-Gal4 intersectional approach (Dionne et al., 2018) to generate a more specific mISEZt line. We took advantage of a second Gal4 line that labeled the same bitter-selective mISEZt neurons as R29F12-Gal4 (Figure 8 - Figure Supplement 2A-B). The split-Gal4 approach allows us to exclusively label neurons present in both expression patterns, resulting in a highly specific mISEZt line (Figure 8D). We verified that the mISEZt neurons labeled by the split-Gal4 line respond to bitter (Figure 8 - Figure Supplement $2 \mathrm{C}$ ). This line was used for all behavioral experiments described below.

We first tested the role of mISEZt bitter neurons in regulating PER. Activating these neurons using Chrimson suppressed PER to sugar (Figure 8E), similar to the effect of activating bittersensing neurons (Figure 5E). We also tested the effect of neuronal silencing using the light- 
gated chloride channel GTACR (Mohammad et al., 2017). Adding bitter to sugar normally suppresses sugar-induced PER, but silencing the mISEZt bitter neurons reduced this suppression (Figure 8F). Thus, mISEZt bitter neurons partially mediate the aversive effect of bitter on proboscis extension.

In contrast to their effect on proboscis extension, activating mISEZt bitter neurons generally was not sufficient to elicit locomotor changes, innate positional aversion, or learned aversion (Figure 8G-I and Figure 8 - Figure Supplement 3). The only statistically significant effect in any of these behavioral assays, at any light intensity, was an increase in turning at the onset of high intensity light (Figure 8G). Medium intensity light also elicited a similar but non-significant effect (Figure $8 \mathrm{G}$ ). Changes in forward velocity at light onset or offset did not differ from controls (Figure $8 \mathrm{G}$ and Figure 8 - Figure Supplement 3A-B).

Together, these data show that second-order mISEZt bitter neurons regulate a subset of the aversive behaviors elicited by bitter-sensing neurons. Behaviors that were not significantly affected by mISEZt activation may be mediated by other second-order bitter pathways, or they may require the activation of multiple second-order pathways.

\section{DISCUSSION}

In this study we investigated how diverse taste inputs within a single taste modality contribute to behavior. We first showed that optogenetic activation of sugar- or bitter-sensing neurons elicits opposing effects on locomotion, positional preference, and associative learning. We then activated subsets of bitter neurons in different organs and found that they elicited broad and highly overlapping effects on behavior, suggesting that these inputs activate common downstream pathways. However, there were some behavioral differences that suggest that these inputs are not integrated in a fully unbiased manner. Consistent with these results, transsynaptic tracing suggested that bitter neuron subsets connect to overlapping second-order pathways, but different subsets preferentially connect to different pathways. We confirmed that one type of second-order neuron, mISEZt, receives bitter input from multiple organs. mISEZt bitter neurons project to the SLP and regulate proboscis extension, but have little to no effect on other bitter-induced behaviors. These results show that within a taste modality, different inputs are selectively integrated early in the circuit and activate multiple downstream pathways, which may make different contributions to behavior.

\section{Behavioral effects of optogenetic taste activation}

We used optogenetic activation to test the behavioral roles of taste sensory neurons. Activating sugar neurons suppressed locomotion and elicited innate and learned preference, whereas activating bitter neurons enhanced locomotion and elicited innate and learned aversion. These results reproduce the known effects of natural sugar or bitter (Flood et al., 2013; Thoma et al., 2016; Marella et al., 2006; Joseph and Heberlein, 2012; Das et al., 2014; Schwaerzel et al., 2003), providing reassurance that our activation paradigms are relevant to natural behavior. Our 
results also align with other studies that reported effects on locomotion or innate preference using optogenetic taste neuron activation (Aso et al., 2014; Tadres and Louis, 2020; Mohammad et al., 2017; Haberkern et al., 2019; DeAngelis et al., 2020; Stern and Yang, 2017).

One counterintuitive result was our finding that the positional attraction elicited by sugar neuron activation diminishes and switches to avoidance with high intensity stimulation, an observation also noted in a previous study (Mohammad et al., 2017). With natural sugar, positional preference increases with concentration even up to very high concentrations such as $2 \mathrm{M}$ sucrose (Thoma et al., 2016). The optogenetically induced avoidance may therefore result from unnatural levels or patterns of sugar neuron activation, which may cause compensatory changes in the downstream circuit. However, a recent study showed that activating different subsets of sugar neurons at the same relatively low intensity $\left(6.8 \mu \mathrm{W} / \mathrm{mm}^{2}\right)$ elicited different effects on positional preference, including avoidance (Chen et al., 2022). Avoidance of sugar may be relevant during specific behaviors such as egg-laying, as flies avoid laying eggs on sugar in some contexts (Yang et al., 2008; Chen et al., 2022).

We found that all of the behavioral effects of sugar neuron activation were strongly modulated or, in some cases, strictly gated by hunger state. This contrasts with the minimal effect of hunger on most behaviors induced by bitter neuron activation. We expected that bitter-induced behaviors might be partially suppressed by starvation, as has been shown for the effect of bitter on proboscis extension (Inagaki et al., 2014; LeDue et al., 2016). This suppression is thought to be adaptive because it encourages starving flies to be "less choosy" and feed on food sources even if they are contaminated with bitter compounds. However, we did not observe any suppression of bitter-induced behaviors by starvation. In fact, the one effect of starvation was an enhancement of learned aversion. These results argue that different behaviors elicited by the same taste may be differentially gated by hunger. To achieve this selective modulation, hungerdependent gating must occur downstream of sensory neurons. Previous studies have identified hunger modulation of bitter sensory neuron responses (Inagaki et al., 2014; LeDue et al., 2016), but additional modulation may occur in the downstream circuit.

The differential gating of sugar- and bitter-induced behaviors by hunger state aligns with the ethological relevance of each taste modality. Sweet taste promotes caloric intake, which is only necessary when energy stores are low. Bitter taste prevents the ingestion of toxins, which is important whether or not an animal is food-deprived. When we simultaneously activated sugar and bitter neurons, the effects induced by bitter activation dominated over those induced by sugar activation. In most cases the dominance was complete: activating both types of neurons together elicited the same level of locomotion or avoidance as activating bitter neurons alone. The taste circuit likely evolved this strict hierarchy due to the potentially deadly consequences of ingesting toxins; preventing such an outcome generally should take precedence over caloric consumption. How this non-linear integration occurs at the circuit level will be an interesting question for future study. 


\section{Roles of bitter neuron subsets}

Conventional models have proposed that taste neurons in different organs have distinct behavioral roles (Dethier, 1976; Scott, 2018). We were therefore surprised to find that activating subsets of bitter neurons in three different organs, the leg, labellum, and pharynx, had broad and largely overlapping effects on behavior. Activating neurons in any of the three organs enhanced locomotor speed and turning, elicited innate aversion, and suppressed proboscis extension. Neurons in the leg and labellum also elicited learned aversion. These findings suggest that bitter inputs from different organs converge in the brain to regulate a common set of aversive behaviors. We have not performed a similar set of experiments for sugar neurons, and it is possible that they would show a greater degree of organ-specific behavioral regulation. Indeed, several studies have shown that sugar neurons in different organs have different behavioral effects (Thoma et al., 2016; Murata et al., 2017; Chen et al., 2021; Chen et al., 2022). Perhaps organ-specific roles for appetitive tastes are needed to generate the appropriate sequence of motor subprograms during feeding, whereas aversive behaviors do not follow an organ-specific sequence.

However, the effects of activating different bitter neuron subsets were not identical. For example, activating pharyngeal bitter neurons elicited stronger innate aversion than leg or labellar (S-a + I-a) neurons, but the latter two subsets elicited stronger learned aversion than pharyngeal neurons (Figures 5 and 6). Differences like these cannot be explained by technical issues such as the number of neurons activated or the strength of Gal4 expression. These results argue against a model in which all bitter inputs converge in an unbiased manner onto downstream behavioral pathways. Instead, downstream pathways may selectively integrate different bitter inputs.

We were also curious whether different subsets of bitter neurons within the same organ would show similar or distinct behavioral effects. Due to limitations in driver lines available when we initiated this study, thus far we have only compared two such subsets: labellar neurons belonging to the S-a + I-a classes versus the S-b class. S-a + I-a neurons drove aversive effects in all behavioral assays tested, whereas S-b neurons had a much more limited effect. However, there are fewer S-b neurons (3) than S-a + I-a neurons (13) and the Gal4 line used to label S-b neurons was expressed more weakly, limiting our interpretation of these results. A recent study showed that two types of pharyngeal bitter neurons regulate different aspects of feeding behavior (Chen et al., 2019), supporting the notion of class-specific behavioral effects.

In general, the existence of multiple bitter neuron classes in each organ, with different tuning profiles (Weiss et al., 2011), would seem to imply that they have different functional roles. If different classes drive distinct aversive behaviors, this would enable the fly to respond differently depending on which bitter compound is detected. Alternatively, input from different classes may be combinatorially combined to encode bitter identity. In this case, different compounds could elicit different behavioral responses without each class having a clearly segregated behavioral role. 


\section{Downstream taste pathways}

Through transsynaptic tracing experiments, we identified three major second-order pathways that relay bitter input to higher brain areas, which have also been described in other recent studies (Chen et al., 2019; Snell et al., 2020). We found that each anatomical pathway receives input from multiple organs, although our experiments do not confirm that different inputs converge onto the same individual cells. Bitter neurons in the labellum provide input onto all three projection tracts, whereas tarsal bitter neurons primarily project onto the lateral and mediolateral tracts and pharyngeal bitter neurons primarily innervate the medial tract. This biased connectivity pattern may provide a neuronal substrate for our behavioral results: different subsets of bitter neurons elicit overlapping but non-identical behavioral effects because they activate overlapping but non-identical second-order taste pathways.

Bitter-sensing neurons in different organs show striking differences in their axonal projection patterns in the SEZ (Wang et al., 2004; Kwon et al., 2014). We were therefore surprised that our trans-Tango experiments did not reveal any obvious organ-specific projection pathways. However, local organ-specific pathways may exist within the SEZ, whereas our study focused on second-order pathways projecting out of the SEZ. In the sugar-sensing circuit, several organspecific downstream pathways have been identified. IN1 interneurons in the SEZ receive sugar input specifically from the pharynx and regulate sugar ingestion (Yapici et al., 2016). A set of sugar-responsive serotonergic neurons in the SEZ receives input from the labellum, but not the leg, and regulates sugar intake and insulin release (Yao and Scott, 2022). Finally, some tarsal sugar-sensing neurons arborize in the ventral nerve cord (Kwon et al., 2014) and activate legspecific ascending second-order neurons (Kim et al., 2017), one of which regulates egg-laying behavior (Chen et al., 2022).

The second-order bitter neurons we characterized, mISEZt neurons, do not appear to be organspecific. mISEZt neurons are activated by bitter tastants presented on either the leg or labellum, demonstrating that bitter information from multiple organs is integrated at the first layer of the taste circuit. It will be interesting to compare bitter encoding across different second-order neurons. Different second-order pathways may convey different features of the taste stimulus, perhaps analogous to the parallel "what" and "where" pathways in the visual and auditory systems (Goodale and Milner, 1992; Lee, 2013). For example, different second-order pathways may transmit the bitter ON versus OFF response, thus encoding the start and end of the stimulus. Certain pathways may retain information about which organ detected the stimulus while others do not. Similarly, some pathways may integrate bitter information with other taste inputs to encode the overall taste valence of the substrate. However, significant transformations of bitter encoding do not seem to occur in mISEZt neurons, as their taste selectivity, response dynamics, and experience-dependent modulation closely resemble responses in sensory neurons.

Do different second-order bitter pathways have different behavioral roles? Activating mISEZt neurons revealed that they regulate proboscis extension but exert little to no effect on locomotion, innate preference, or associative learning. Behaviors not affected by mISEZt 
neurons may be regulated by other pathways, and it is possible that different pathways strictly control different behaviors. This has been suggested in the mammalian taste system, in which a cortico-amygdalar projection regulates licking behavior but is dispensable for reporting the identity of the stimulus in a learned discrimination task (Wang et al., 2018). Similarly, distinct pathways in the olfactory system are thought to mediate innate versus learned odor responses (Bear et al., 2016; Amin and Lin, 2019). Having separate pathways that regulate different behavioral responses allows a circuit to specifically modulate a subset of those behaviors depending on context or state.

Alternatively, different second-order pathways may have overlapping behavioral roles. Perhaps different pathways encode different aspects of the stimulus, as discussed above, and are combined downstream in specific ways appropriate for regulating each behavior. In support of this combinatorial model, we found that different bitter-sensing neurons elicited overlapping behavioral effects even when they had largely non-overlapping projection targets. Tarsal bitter neurons mainly project to the lateral and mediolateral second-order pathways, whereas the pharyngeal bitter neuron mainly projects to the medial pathway, but both subsets elicited strong effects on proboscis extension, locomotor speed, and turning. It therefore seems likely that different second-order pathways may converge downstream to regulate a common set of behaviors.

\section{Integration of sensory inputs}

Within a single taste modality, whether and how different channels of taste input are integrated has been largely unexplored. Our results show that inputs from different bitter neuron types are integrated early in the circuit and drive an overlapping, but not identical, set of behaviors. It will be interesting to determine whether similar principles govern the integration of taste input in other taste modalities and other organisms. Humans and other mammals have taste neurons in multiple locations, including three distinct areas of the tongue, the soft palate, pharynx, and internal organs such as the gut (Liman et al., 2014; Depoortere, 2014; Travers and Nicklas, 1990). Moreover, mammalian bitter-sensing cells in the tongue show heterogeneous responses (Caicedo and Roper, 2001) and express different combinations of bitter receptors (Behrens et al., 2007; Voigt et al., 2012), as in flies, suggesting the presence of different functional classes. Taste may thus provide a model for understanding and comparing how neural circuits integrate different sensory input channels, a question at the core of how we interpret signals from the world. 


\section{MATERIALS AND METHODS}

\section{Key Resources Table}

\begin{tabular}{|c|c|c|c|c|}
\hline $\begin{array}{l}\text { Reagent type } \\
\text { (species) or } \\
\text { resource }\end{array}$ & Designation & Source or reference & Identifiers & $\begin{array}{l}\text { Additional } \\
\text { information }\end{array}$ \\
\hline $\begin{array}{l}\text { genetic reagent ( } D . \\
\text { melanogaster) }\end{array}$ & Gr64f-Gal4 & $\begin{array}{l}\text { Dahanukar et al. } \\
\text { (2007) }\end{array}$ & BDSC: 57669 & \\
\hline $\begin{array}{l}\text { genetic reagent ( } D . \\
\text { melanogaster) }\end{array}$ & Gr33a-Gal4 & Moon et al. (2009) & BDSC: 31425 & \\
\hline $\begin{array}{l}\text { genetic reagent ( } D . \\
\text { melanogaster) }\end{array}$ & Gr58c-Gal4 & Weiss et al. (2011) & BDSC: 57646 & \\
\hline $\begin{array}{l}\text { genetic reagent ( } D . \\
\text { melanogaster) }\end{array}$ & Gr59c-Gal4 & Weiss et al. (2011) & BDSC: 57650 & \\
\hline $\begin{array}{l}\text { genetic reagent ( } D . \\
\text { melanogaster) }\end{array}$ & Gr22f-Gal4 & Weiss et al. (2011) & BDSC: 57610 & \\
\hline $\begin{array}{l}\text { genetic reagent ( } D . \\
\text { melanogaster) }\end{array}$ & Gr9a-Gal4 & Weiss et al. (2011) & BDSC: 57596 & \\
\hline $\begin{array}{l}\text { genetic reagent }(D . \\
\text { melanogaster) }\end{array}$ & R29F12-Gal4 & Jenett et al. (2012) & BDSC: 49495 & \\
\hline $\begin{array}{l}\text { genetic reagent }(D . \\
\text { melanogaster) }\end{array}$ & R55E01-Gal4 & Jenett et al. (2012) & BDSC: 39117 & \\
\hline $\begin{array}{l}\text { genetic reagent ( } D . \\
\text { melanogaster) }\end{array}$ & $R 29 F 12-A D$ & Dionne et al. (2018) & BDSC: 71164 & \\
\hline $\begin{array}{l}\text { genetic reagent ( } D . \\
\text { melanogaster) }\end{array}$ & R55E01-DBD & Dionne et al. (2018) & BDSC: 69662 & \\
\hline $\begin{array}{l}\text { genetic reagent ( } D . \\
\text { melanogaster) }\end{array}$ & Gr66a-lexA & Thistle et al. (2012) & BDSC: 93024 & \\
\hline $\begin{array}{l}\text { genetic reagent ( } D . \\
\text { melanogaster) }\end{array}$ & $\begin{array}{l}\text { UAS-Chrimson- } \\
T d T\end{array}$ & $\begin{array}{l}\text { Duistermars et al. } \\
\text { (2018) }\end{array}$ & $\mathrm{N} / \mathrm{A}$ & \\
\hline $\begin{array}{l}\text { genetic reagent ( } D . \\
\text { melanogaster) }\end{array}$ & $\begin{array}{l}\text { UAS-GTACR1- } \\
T d T\end{array}$ & B. Noro & $\mathrm{N} / \mathrm{A}$ & \\
\hline $\begin{array}{l}\text { genetic reagent ( } D . \\
\text { melanogaster) }\end{array}$ & $\begin{array}{l}\text { trans-Tango } \\
\text { reporter (UAS- } \\
\text { Myr-GFP, QUAS- } \\
\text { mtdTomato; trans- } \\
\text { Tango) }\end{array}$ & Talay et al. (2017) & BDSC: 77124 & \\
\hline $\begin{array}{l}\text { genetic reagent ( } D . \\
\text { melanogaster) }\end{array}$ & UAS-GCaMP6f & Chen et al. (2013) & BDSC: 42747 & \\
\hline $\begin{array}{l}\text { genetic reagent ( } D . \\
\text { melanogaster) }\end{array}$ & UAS-TdTomato & D. Hattori & $\mathrm{N} / \mathrm{A}$ & \\
\hline $\begin{array}{l}\text { genetic reagent ( } D . \\
\text { melanogaster) }\end{array}$ & lexAop-GCaMP6f & $\begin{array}{l}\text { D. Kim; Hattori et al. } \\
(2017)\end{array}$ & BDSC 44277 & \\
\hline antibody & chicken anti-GFP & Aves Labs & $\begin{array}{l}\text { Cat\# GFP-1020; } \\
\text { RRID: } \\
\text { AB_10000240 }\end{array}$ & \\
\hline antibody & rabbit anti-DsRed & Clontech & $\begin{array}{l}\text { Cat\# 632496; RRID: } \\
\text { AB_10013483 }\end{array}$ & \\
\hline antibody & $\begin{array}{l}\text { mouse anti- } \\
\text { bruchpilot (nc82) }\end{array}$ & $\begin{array}{l}\text { Development Studies } \\
\text { Hybridoma Bank }\end{array}$ & $\begin{array}{l}\text { Cat\# nc82; RRID: } \\
\text { AB_2314866 }\end{array}$ & \\
\hline antibody & $\begin{array}{l}\text { Alexa Fluor } 488 \\
\text { goat anti-chicken }\end{array}$ & Life Technologies & $\begin{array}{l}\text { Cat\# A11039; RRID: } \\
\text { AB_2534096 }\end{array}$ & \\
\hline
\end{tabular}




\begin{tabular}{|l|l|l|l|l|}
\hline antibody & $\begin{array}{l}\text { Alexa Fluor 568 } \\
\text { goat anti-rabbit }\end{array}$ & Life Technologies & $\begin{array}{l}\text { Cat\# A11036; RRID: } \\
10563566\end{array}$ & \\
\hline antibody & $\begin{array}{l}\text { Alexa Fluor 633 } \\
\text { goat anti-mouse }\end{array}$ & Life Technologies & $\begin{array}{l}\text { Cat\# A21052; RRID: } \\
\text { AB_2535719 }\end{array}$ & \\
\hline software, algorithm & Prism, version 9 & GraphPad & N/A & \\
\hline software, algorithm & MATLAB & Mathworks & N/A & \\
\hline software, algorithm & FlyTracker & $\begin{array}{l}\text { Caltech; Eyjolfsdottir } \\
\text { et al. (2014) }\end{array}$ & N/A & $\begin{array}{l}\text { http://www.vision. } \\
\text { caltech.edu/Tools/ } \\
\text { FlyTracker/ }\end{array}$ \\
\hline
\end{tabular}

\section{Fly stocks and maintenance}

Flies were reared at $25^{\circ}$ on standard cornmeal food, with the exception of flies for trans-Tango experiments which were reared at $20^{\circ}$. All transgenic lines used for behavior, with the exception of the split-Gal4 line, were outcrossed into a $2 U$ wild-type background for at least 5 generations. Experiments were performed on mated females, with the exception of trans-Tango experiments that used males (the location of transgenes on the $\mathrm{X}$ chromosome can lead to enhanced expression in males). Behavioral assays were performed on 3-7 day-old flies, calcium imaging was performed on 2-3 week-old flies (to ensure robust GCaMP expression), and trans-Tango staining was performed on $\sim 4$ week-old flies (to ensure robust signal). Flies used for optogenetic experiments were maintained in constant darkness and fed on food containing 1 $\mathrm{mM}$ all trans-retinal for 3-5 days prior to testing. For optogenetic experiments using starved flies, flies were food-deprived with water for the specified duration using a wet piece of Kimwipe containing all $1 \mathrm{mM}$ trans-retinal.

\section{Locomotor and preference assays}

The behavioral arena for testing locomotor and preference behaviors was built based on designs by the Rubin lab at the Janelia Research Campus (Aso et al., 2014; Aso and Rubin, 2016). The arena contains a circular chamber with a glass cover, and flies are filmed from above using a USB camera (Point Grey). The infrared light for illumination and the red (627 $\mathrm{nm}$ ) LED array for optogenetic stimulation are located just beneath the chamber. Each quadrant of the arena has an inlet for air flow, allowing for odor delivery, and air is removed through a vacuum port at the center of the arena. We did not use air flow for locomotor or innate preference assays. 25-30 flies were tested per trial. Flies were loaded into the chamber using mouth aspiration and were given at least 3 minutes to habituate before the experiment started. Flies were filmed at 30 frames/second.

For learning experiments using odor, $400 \mathrm{~mL} / \mathrm{min}$ air was split into two streams that each flowed to one pair of opposing quadrants and could be odorized independently. Each stream was odorized by flowing air through bottles containing odor dilutions. Each stream was then split again to provide input to two opposing quadrants, representing a flow rate of $100 \mathrm{~mL} / \mathrm{min}$ air into each quadrant. The odors used were 3-octanol (1:1000) and 4-methylcyclohexanol (1:750). Odors were diluted in mineral oil and $2 \mathrm{~mL}$ of each odor solution was pipetted onto a piece of 
thick filter paper (Thermo Scientific, \#88615) placed inside of a glass odor bottle (Cole-Parmer, \#EW-99535-16).

The effects of taste neuron activation on locomotion and innate preference were assayed sequentially in the same flies. To quantify locomotor effects, light stimulation was presented for $5 \mathrm{sec}$. Flies were filmed for $>1 \mathrm{~min}$ before stimulation and $2 \mathrm{~min}$ after stimulation, although only the $5 \mathrm{sec}$ prior to stimulation was used to quantify baseline behavior. Each of the three light intensities was delivered to the same flies in increasing order of intensity, with several minutes between stimulations to ensure that the behavior had recovered. After another rest period, the same flies were then tested for innate preference by delivering low intensity light stimulation to two opposing quadrants for $30 \mathrm{sec}$. The flies then had a $30 \mathrm{sec}$ rest period without light, followed by low intensity light stimulation in the other two quadrants for $30 \mathrm{sec}$. Switching which quadrants the light was presented in ensured that we could assess light preference independently of any spatial bias the flies may have. This protocol was then repeated sequentially with medium and high intensity light.

To test learned odor preference, the CS+ odor was presented in all quadrants for 1 min along with light stimulation. Light stimulation started $5 \mathrm{sec}$ after odor onset and was delivered in $1 \mathrm{~Hz}$ pulses, following the protocol used in previous associative learning studies (Aso and Rubin, 2016). After a $1 \mathrm{~min}$ rest, the CS- odor was then presented alone for $1 \mathrm{~min}$. Following a $1 \mathrm{~min}$ rest period, the CS+ and CS- odors were simultaneously delivered to different sets of opposing quadrants for $1 \mathrm{~min}$, allowing the flies to choose between the odors. After another $1 \mathrm{~min}$ rest, the two odors were presented again for $1 \mathrm{~min}$ but the odor quadrants were switched in order to control for any spatial bias. Which odor was used as the CS+ or CS- was counterbalanced across trials. The light intensity used for learning experiments with bitter neuron activation was $30 \mu \mathrm{W} / \mathrm{mm}^{2}$. For sugar neuron activation we decreased the intensity to $4 \mu \mathrm{W} / \mathrm{mm}^{2}$ because this was the intensity at which sugar neurons elicited innate preference.

Fly movies were analyzed using FlyTracker (Eyjolfsdottir et al., 2014), which quantified the position, forward velocity, and angular velocity of each fly at each time point. FlyTracker output was further analyzed in MATLAB to quantify preference for light or odor and to generate the traces shown in the figures. Preference index (PI) values were quantified in $1 \mathrm{sec}$ bins and calculated as (\# flies in light quadrants - \# flies in non-light quadrants) / total \# flies. PI for the conditioned odor was calculated similarly as (\# flies in CS+ quadrants - \# flies in CSquadrants) / total \# flies. Forward and angular velocities were averaged over $0.33 \mathrm{sec}$ bins (10 frames). To quantify locomotor changes at light onset, we averaged forward or angular velocity over the first $1 \mathrm{sec}$ or entire $5 \mathrm{sec}$ of light presentation (specified in the figure legends) and compared these values to baseline values averaged over the $4 \mathrm{sec}$ preceding light onset. To quantify the change in speed after light stimulation, we averaged forward velocity over a $5 \mathrm{sec}$ period following light offset and compared it to the same baseline (pre-light) value described above. For statistical analyses of these data, each trial was considered to be a single data point (" $n$ "). Further analyses are described below in the "Statistical analyses" section. 


\section{PER assay}

PER was tested using previously described methods (Devineni et al., 2019). Briefly, flies were anesthetized on ice, immobilized on their backs with myristic acid, and the two anterior pairs of legs were glued down so that the labellum was accessible for taste stimulation. Flies recovered from gluing for 30-60 min in a humidified chamber. Flies were water-satiated before testing. Tastants were briefly applied to the labellum using a small piece of Kimwipe. For optogenetic stimulation, a $1 \mathrm{sec}$ red or green LED was manually turned on just before the taste stimulus and remained on for the duration of the stimulus. $\sim 10$ flies were sequentially tested in each experiment, and the percent of flies showing PER to each stimulus was manually recorded. Only full proboscis extensions were counted as PER. At the end of each experiment, flies were tested with a positive control (500 mM sucrose) and were excluded from analysis if they did not respond. For statistical analyses, each set of $\sim 10$ flies was considered to be a single data point ("n").

To test bitter neuron suppression of PER, flies were stimulated with sucrose at $100 \mathrm{mM}$ (Figure $5 \mathrm{E}$ ) or $50 \mathrm{mM}$ (Figure 8E); we changed the concentration because these experiments were conducted at different times and the baseline behavior to sucrose had slightly shifted. To test PER suppression by natural bitter, $10 \mathrm{mM}$ quinine was added to $50 \mathrm{mM}$ sucrose (Figure $8 \mathrm{~F}$ ).

\section{Immunohistochemistry}

Immunostaining experiments were performed as previously described (Devineni et al., 2021). Briefly, brains were dissected in phosphate buffered saline (PBS), fixed for $15-20 \mathrm{~min}$ in $4 \%$ paraformaldehyde, washed multiple times with PBS containing 0.3\% Triton X-100 (PBST), blocked with $5 \%$ normal goat serum for $1 \mathrm{hr}$, incubated with primary antibodies at $4^{\circ}$ for $2-3$ days, washed in PBST, incubated with secondary antibodies at $4^{\circ}$ overnight, washed in PBST and PBS, and mounted in Vectashield. Primary antibodies used were chicken anti-GFP (1:1000), rabbit anti-DsRed (1:500), and mouse nc82 (1:10). Secondary antibodies used were Alexa Fluor 488 goat anti-chicken (1:500), Alexa Fluor 568 goat anti-rabbit (1:500), and Alexa Fluor 633 goat anti-mouse (1:500). Images were acquired on a Zeiss LSM 710 system. Confocal images, whether obtained from our own experiments or from the Janelia FlyLight database (https://flweb.janelia.org/cgi-bin/flew.cgi), were processed using Fiji.

\section{Calcium imaging}

Calcium imaging was performed as previously described (Devineni et al., 2019; Devineni et al., 2021). Flies were taped on their backs to a piece of clear tape in an imaging chamber. For proboscis taste stimulation, fine strands of tape were used to restrain the legs, secure the head, and immobilize the proboscis in an extended position. For leg taste stimulation, the two forelegs were immobilized using tape and parafilm with the distal segment exposed. An imaging window was cut on the anterior surface of the head, the antennae were removed, and the esophagus was cut. The brain was immersed in modified artificial hemolymph in which $15 \mathrm{mM}$ ribose is substituted for sucrose and trehalose (Marella et al., 2006; Wang et al., 2003). 
Images were acquired using a two-photon laser scanning microscope (Ultima, Bruker) equipped with an ultra-fast Ti:S laser (Chameleon Vision, Coherent) that is modulated by pockel cells (Conoptics). The excitation wavelength was $925 \mathrm{~nm}$. Emitted photons were collected with a GaAsP photodiode detector (Hamamatsu) through a 60X water-immersion objective (Olympus). Images were acquired using the microscope software (PrairieView, Bruker). A single plane was chosen for imaging in each experiment. For most experiments, images were acquired at 256 by 256 pixels with a scanning rate of 3-4 Hz. The resolution was decreased and scanning rate was increased to $\sim 6-7 \mathrm{~Hz}$ for experiments using repeated taste stimulation.

Tastants were delivered to the labellum or foreleg as previously described (Devineni et al., 2019; Devineni et al., 2021). Briefly, we used a custom-built solenoid pinch valve system controlled by MATLAB software via a data acquisition device (Measurement Computing). Solenoid pinch valves (Cole Parmer) were opened briefly ( $10 \mathrm{~ms})$ to create a small liquid drop at the end of a $5 \mu \mathrm{L}$ glass capillary, positioned such that the drop would make contact with the labellum or leg. Tastants were removed by a vacuum line controlled by a solenoid pinch valve. Proper taste delivery was monitored using a side-mounted camera (Veho VMS-004). 5 sec taste stimulation was used for all experiments. At least three trials of each stimulus were given to each fly. Other than experiments explicitly using repeated taste stimulation, at least one minute rest was given between trials to avoid habituation. Tastants were used at the following concentrations: $10 \mathrm{mM}$ quinine, $10 \mathrm{mM}$ denatonium, $100 \mathrm{mM}$ caffeine, $25 \mathrm{mM}$ L-canavanine, and $100 \mathrm{mM}$ sucrose.

Calcium imaging data were analyzed using MATLAB code from previous studies (Devineni et al., 2019; Devineni et al., 2021). Images were registered within and across trials to correct for movement in the $x-y$ plane using a sub-pixel registration algorithm. Regions of interest (ROls) were drawn manually. Average pixel intensity within the ROI was calculated for each frame. The average signal for 20 frames preceding stimulus delivery was used as the baseline signal, and the $\Delta F / F$ (change in fluorescence divided by baseline) for each frame was then calculated.

\section{Statistical analyses}

Statistical analyses were performed using GraphPad Prism, Version 9. Statistical tests and results are reported in the figure legends. Two groups were compared using unpaired t-tests. More than two groups were compared using one-way ANOVA followed by Dunnett's test comparing experimental flies to each control. All graphs represent mean \pm SEM. Sample sizes are listed in the figure legends.

To generate the table in Figure 4A, the effects of sugar and bitter neuron activation were analyzed separately. For each genotype and behavioral parameter, one-way ANOVAs were used to determine if there was an effect at any light intensity. If so, the relative strength of the effect was quantified for each starvation condition by comparing the maximal effect at any intensity to the maximal effect observed for a given neuron type (sugar or bitter) across all conditions and light intensities. To do this, the difference between experimental flies and each 
control genotype was quantified, and the smaller of the two differences was used as a measure of the experimental effect. A strong or moderate effect was defined as an effect at least $80 \%$ or $40 \%$, respectively, of the maximal value observed under any starvation condition. Statistically significant effects that were less than $40 \%$ of the maximal value were defined as weak effects. For sugar-sensing neurons, only the attractive innate preference induced by low light intensities was analyzed; behavior at higher light intensities is not considered here. To best capture the effects observed, light-induced locomotion was quantified over the entire $5 \mathrm{sec}$ period for sugar neurons but only over the first $1 \mathrm{sec}$ for bitter neurons.

The table in Figure 6 was generated using the same methods as for Figure 4A, except that the effect of activating each bitter neuron subset was compared to the maximal effect observed when activating all bitter neurons.

\section{ACKNOWLEDGEMENTS}

We thank Richard Axel for his generous support and helpful discussions. We also thank Katie Shakman for initial setup of the behavioral arena and some analysis code; Tanya Tabachnik and Rick Hormigo for technical assistance with the behavioral arena; Barbara Noro for sharing unpublished fly lines, general advice, and manuscript feedback; Yu-Chieh David Chen and Chris Rodgers for comments on the manuscript; and John Carlson, Gilad Barnea, the Janelia Research Center, and the Bloomington Drosophila Stock Center (BDSC) for providing fly strains.

\section{AUTHOR CONTRIBUTIONS}

A.V.D conceived and supervised the project. J.U.D., H.A.U., and N.M.S conducted behavioral experiments; J.U.D. performed brain dissections and immunostaining; A.V.D. conducted calcium imaging experiments. A.V.D generated figures and wrote the manuscript with input from all authors. 


\section{REFERENCES}

Abraira VE, and Ginty DD. 2013. The sensory neurons of touch. Neuron 79:618-639.

Amin H, and Lin AC. 2019. Neuronal mechanisms underlying innate and learned olfactory processing in Drosophila. Curr Opin Insect Sci 36:9-17.

Aso Y, and Rubin GM. 2016. Dopaminergic neurons write and update memories with cell-typespecific rules. Elife 5 .

Aso Y, Sitaraman D, Ichinose T, Kaun KR, Vogt K, Belliart-Guerin G, Placais PY, Robie AA, Yamagata N, Schnaitmann C, et al. 2014. Mushroom body output neurons encode valence and guide memory-based action selection in Drosophila. Elife 3:e04580.

Bates AS, Schlegel P, Roberts RJV, Drummond N, Tamimi IFM, Turnbull R, Zhao X, Marin EC, Popovici PD, Dhawan S, et al. 2020. Complete connectomic reconstruction of olfactory projection neurons in the fly brain. Curr Biol 30:3183-3199 e3186.

Bear DM, Lassance JM, Hoekstra HE, and Datta SR. 2016. The evolving neural and genetic architecture of vertebrate olfaction. Curr Biol 26:R1039-R1049.

Behrens M, Foerster S, Staehler F, Raguse JD, and Meyerhof W. 2007. Gustatory expression pattern of the human tas $2 r$ bitter receptor gene family reveals a heterogenous population of bitter responsive taste receptor cells. J Neurosci 27:12630-12640.

Caicedo A, and Roper SD. 2001. Taste receptor cells that discriminate between bitter stimuli. Science 291:1557-1560.

Chen HL, Motevalli D, Stern U, and Yang CH. 2022. A functional division of Drosophila sweet taste neurons that is value-based and task-specific. Proc Natl Acad Sci U S A 119.

Chen YD, and Dahanukar A. 2017. Molecular and cellular organization of taste neurons in adult Drosophila pharynx. Cell Rep 21:2978-2991.

Chen YD, Menon V, Joseph RM, and Dahanukar AA. 2021. Control of sugar and amino acid feeding via pharyngeal taste neurons. J Neurosci 10.1523/JNEUROSCI.1794-20.2021.

Chen YD, Park SJ, Joseph RM, Ja WW, and Dahanukar AA. 2019. Combinatorial pharyngeal taste coding for feeding avoidance in adult Drosophila. Cell Rep 29:961-973 e964.

Dahanukar A, Lei YT, Kwon JY, and Carlson JR. 2007. Two gr genes underlie sugar reception in Drosophila. Neuron 56:503-516.

Das G, Klappenbach M, Vrontou E, Perisse E, Clark CM, Burke CJ, and Waddell S. 2014. Drosophila learn opposing components of a compound food stimulus. Curr Biol 24:1723-1730.

DeAngelis BD, Zavatone-Veth JA, Gonzalez-Suarez AD, and Clark DA. 2020. Spatiotemporally precise optogenetic activation of sensory neurons in freely walking Drosophila. Elife 9.

Depoortere I. 2014. Taste receptors of the gut: Emerging roles in health and disease. Gut 63:179-190. 
Dethier V (1976). The hungry fly (Cambridge: Harvard University Press).

Devineni AV, Deere JU, Sun B, and Axel R. 2021. Individual bitter-sensing neurons in Drosophila exhibit both on and off responses that influence synaptic plasticity. Curr Biol 31:5533-5546 e5537.

Devineni AV, Sun B, Zhukovskaya A, and Axel R. 2019. Acetic acid activates distinct taste pathways in Drosophila to elicit opposing, state-dependent feeding responses. Elife 8.

Dionne H, Hibbard KL, Cavallaro A, Kao JC, and Rubin GM. 2018. Genetic reagents for making split-GAL4 lines in Drosophila. Genetics 209:31-35.

Duistermars BJ, Pfeiffer BD, Hoopfer ED, and Anderson DJ. 2018. A brain module for scalable control of complex, multi-motor threat displays. Neuron 100:1474-1490 e1474.

Estebanez L, Ferezou I, Ego-Stengel V, and Shulz DE. 2018. Representation of tactile scenes in the rodent barrel cortex. Neuroscience 368:81-94.

Eyjolfsdottir E, Branson S, Burgos-Artizzu XP, Hoopfer ED, Schor J, Anderson DJ, and Perona $P$ (2014). Detecting social actions of fruit flies (Cham: Springer International Publishing).

Flood TF, Iguchi S, Gorczyca M, White B, Ito K, and Yoshihara M. 2013. A single pair of interneurons commands the Drosophila feeding motor program. Nature 499:83-87.

Fujii S, Yavuz A, Slone J, Jagge C, Song X, and Amrein H. 2015. Drosophila sugar receptors in sweet taste perception, olfaction, and internal nutrient sensing. Curr Biol 25:621-627.

Goodale MA, and Milner AD. 1992. Separate visual pathways for perception and action. Trends Neurosci 15:20-25.

Haberkern H, Basnak MA, Ahanonu B, Schauder D, Cohen JD, Bolstad M, Bruns C, and Jayaraman V. 2019. Visually guided behavior and optogenetically induced learning in headfixed flies exploring a virtual landscape. Curr Biol 29:1647-1659 e1648.

Hattori D, Aso Y, Swartz KJ, Rubin GM, Abbott LF, and Axel R. 2017. Representations of novelty and familiarity in a mushroom body compartment. Cell 169:956-969 e917.

Haverkamp A, Hansson BS, and Knaden M. 2018. Combinatorial codes and labeled lines: How insects use olfactory cues to find and judge food, mates, and oviposition sites in complex environments. Front Physiol 9:49.

Huetteroth W, Perisse E, Lin S, Klappenbach M, Burke C, and Waddell S. 2015. Sweet taste and nutrient value subdivide rewarding dopaminergic neurons in Drosophila. Curr Biol 25:751758.

Inagaki HK, Ben-Tabou de-Leon S, Wong AM, Jagadish S, Ishimoto H, Barnea G, Kitamoto T, Axel R, and Anderson DJ. 2012. Visualizing neuromodulation in vivo: Tango-mapping of dopamine signaling reveals appetite control of sugar sensing. Cell 148:583-595.

Inagaki HK, Panse KM, and Anderson DJ. 2014. Independent, reciprocal neuromodulatory control of sweet and bitter taste sensitivity during starvation in Drosophila. Neuron 84:806-820. 
Jenett A, Rubin GM, Ngo TT, Shepherd D, Murphy C, Dionne H, Pfeiffer BD, Cavallaro A, Hall D, Jeter J, et al. 2012. A GAL4-driver line resource for Drosophila neurobiology. Cell Rep 2:9911001.

Jin H, Fishman ZH, Ye M, Wang L, and Zuker CS. 2021. Top-down control of sweet and bitter taste in the mammalian brain. Cell 184:257-271 e216.

Joseph RM, and Heberlein U. 2012. Tissue-specific activation of a single gustatory receptor produces opposing behavioral responses in Drosophila. Genetics 192:521-532.

Joseph RM, Sun JS, Tam E, and Carlson JR. 2017. A receptor and neuron that activate a circuit limiting sucrose consumption. Elife 6.

Kim H, Kirkhart C, and Scott K. 2017. Long-range projection neurons in the taste circuit of Drosophila. Elife 6.

King AJ, Teki S, and Willmore BDB. 2018. Recent advances in understanding the auditory cortex. F1000Res 7.

Klapoetke NC, Murata Y, Kim SS, Pulver SR, Birdsey-Benson A, Cho YK, Morimoto TK, Chuong AS, Carpenter EJ, Tian Z, et al. 2014. Independent optical excitation of distinct neural populations. Nat Methods 11:338-346.

Kwon JY, Dahanukar A, Weiss LA, and Carlson JR. 2014. A map of taste neuron projections in the Drosophila cns. J Biosci 39:565-574.

LeDue EE, Chen YC, Jung AY, Dahanukar A, and Gordon MD. 2015. Pharyngeal sense organs drive robust sugar consumption in Drosophila. Nat Commun 6:6667.

LeDue EE, Mann K, Koch E, Chu B, Dakin R, and Gordon MD. 2016. Starvation-induced depotentiation of bitter taste in Drosophila. Curr Biol 26:2854-2861.

Lee CC. 2013. Thalamic and cortical pathways supporting auditory processing. Brain Lang 126:22-28.

Liman ER, Zhang YV, and Montell C. 2014. Peripheral coding of taste. Neuron 81:984-1000.

Ling F, Dahanukar A, Weiss LA, Kwon JY, and Carlson JR. 2014. The molecular and cellular basis of taste coding in the legs of Drosophila. J Neurosci 34:7148-7164.

Mahishi D, and Huetteroth W. 2019. The prandial process in flies. Curr Opin Insect Sci 36:157166.

Marella S, Fischler W, Kong P, Asgarian S, Rueckert E, and Scott K. 2006. Imaging taste responses in the fly brain reveals a functional map of taste category and behavior. Neuron 49:285-295.

Marella S, Mann K, and Scott K. 2012. Dopaminergic modulation of sucrose acceptance behavior in Drosophila. Neuron 73:941-950. 
Mohammad F, Stewart JC, Ott S, Chlebikova K, Chua JY, Koh TW, Ho J, and Claridge-Chang A. 2017. Optogenetic inhibition of behavior with anion channelrhodopsins. Nat Methods 14:271274.

Moon SJ, Lee Y, Jiao Y, and Montell C. 2009. A Drosophila gustatory receptor essential for aversive taste and inhibiting male-to-male courtship. Curr Biol 19:1623-1627.

Moreira JM, Itskov PM, Goldschmidt D, Baltazar C, Steck K, Tastekin I, Walker SJ, and Ribeiro C. 2019. Optopad, a closed-loop optogenetics system to study the circuit basis of feeding behaviors. Elife 8.

Murata S, Brockmann A, and Tanimura T. 2017. Pharyngeal stimulation with sugar triggers local searching behavior in Drosophila. J Exp Biol 220:3231-3237.

Musso PY, Junca P, Jelen M, Feldman-Kiss D, Zhang H, Chan RC, and Gordon MD. 2019. Closed-loop optogenetic activation of peripheral or central neurons modulates feeding in freely moving Drosophila. Elife 8:e45636.

Rompani SB, Mullner FE, Wanner A, Zhang C, Roth CN, Yonehara K, and Roska B. 2017. Different modes of visual integration in the lateral geniculate nucleus revealed by single-cellinitiated transsynaptic tracing. Neuron 93:767-776 e766.

Rushton WA. 1972. Pigments and signals in colour vision. J Physiol 220:1P-P.

Schwaerzel M, Monastirioti M, Scholz H, Friggi-Grelin F, Birman S, and Heisenberg M. 2003. Dopamine and octopamine differentiate between aversive and appetitive olfactory memories in Drosophila. J Neurosci 23:10495-10502.

Scott K. 2018. Gustatory processing in Drosophila melanogaster. Annu Rev Entomol 63:15-30.

Snell NJ, Fisher JD, Hartmann GG, Talay M, and Barnea G. 2020. Distributed representation of taste quality by second-order gustatory neurons in Drosophila. bioRxiv 10.1101/2020.11.10.377382.

Stern U, and Yang C-H. 2017. Skinnertrax: High-throughput behavior-dependent optogenetic stimulation of Drosophila. bioRxiv.

Tadres D, and Louis M. 2020. Pivr: An affordable and versatile closed-loop platform to study unrestrained sensorimotor behavior. PLoS Biol 18:e3000712.

Talay M, Richman EB, Snell NJ, Hartmann GG, Fisher JD, Sorkac A, Santoyo JF, Chou-Freed C, Nair N, Johnson M, et al. 2017. Transsynaptic mapping of second-order taste neurons in flies by trans-tango. Neuron 96:783-795 e784.

Thistle R, Cameron P, Ghorayshi A, Dennison L, and Scott K. 2012. Contact chemoreceptors mediate male-male repulsion and male-female attraction during Drosophila courtship. Cell 149:1140-1151.

Thoma V, Knapek S, Arai S, Hartl M, Kohsaka H, Sirigrivatanawong P, Abe A, Hashimoto K, and Tanimoto H. 2016. Functional dissociation in sweet taste receptor neurons between and within taste organs of Drosophila. Nat Commun 7:10678. 
Thoma V, Kobayashi K, and Tanimoto H. 2017. The role of the gustatory system in the coordination of feeding. eNeuro 4.

Travers SP, and Nicklas K. 1990. Taste bud distribution in the rat pharynx and larynx. Anat Rec 227:373-379.

Voigt A, Hubner S, Lossow K, Hermans-Borgmeyer I, Boehm U, and Meyerhof W. 2012. Genetic labeling of tas1r1 and tas2r131 taste receptor cells in mice. Chem Senses 37:897-911.

Wang JW, Wong AM, Flores J, Vosshall LB, and Axel R. 2003. Two-photon calcium imaging reveals an odor-evoked map of activity in the fly brain. Cell 112:271-282.

Wang L, Gillis-Smith S, Peng Y, Zhang J, Chen X, Salzman CD, Ryba NJP, and Zuker CS. 2018. The coding of valence and identity in the mammalian taste system. Nature 558:127-131.

Wang Z, Singhvi A, Kong P, and Scott K. 2004. Taste representations in the Drosophila brain. Cell 117:981-991.

Weiss LA, Dahanukar A, Kwon JY, Banerjee D, and Carlson JR. 2011. The molecular and cellular basis of bitter taste in Drosophila. Neuron 69:258-272.

Yang CH, Belawat P, Hafen E, Jan LY, and Jan YN. 2008. Drosophila egg-laying site selection as a system to study simple decision-making processes. Science 319:1679-1683.

Yao Z, and Scott K. 2022. Serotonergic neurons translate taste detection into internal nutrient regulation. Neuron 10.1016/j.neuron.2021.12.028.

Yapici N, Cohn R, Schusterreiter C, Ruta V, and Vosshall LB. 2016. A taste circuit that regulates ingestion by integrating food and hunger signals. Cell 165:715-729. 
A

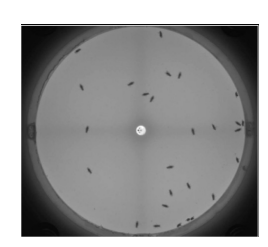

B

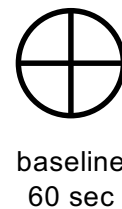

Testing locomotor changes

C
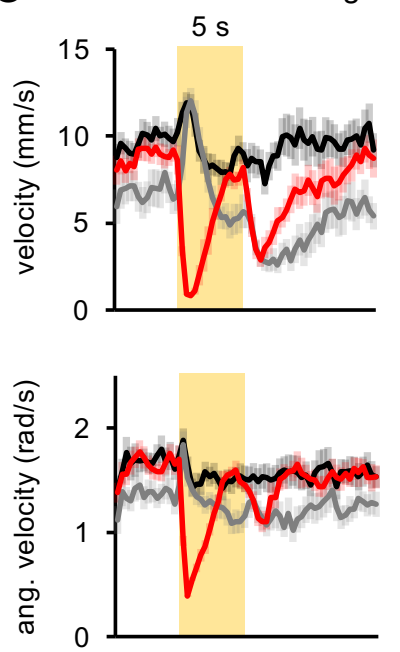

Sugar neuron activation
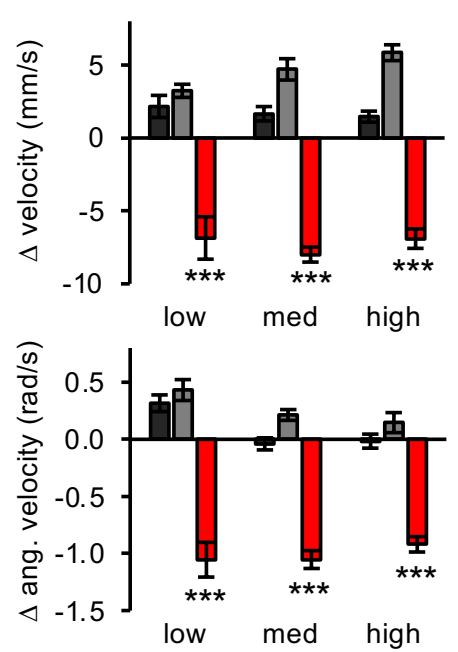

Gr64f-Gal4/+ UAS-Chrim/+ Gr64f-Gal4/UAS-Chrim

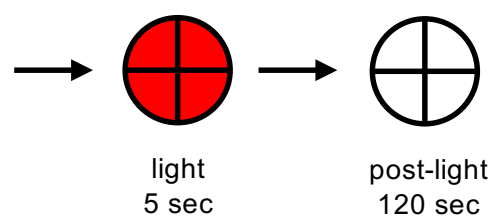

D

Bitter neuron activation
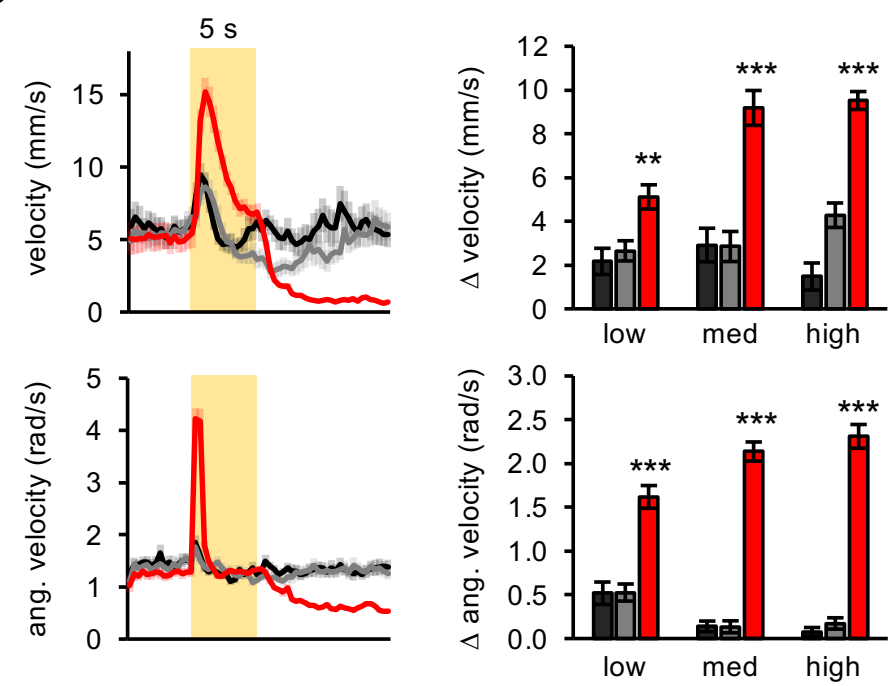

Gr33a-Gal4/+ UAS-Chrim/+ Gr33a-Gal4/UAS-Chrim

$\mathbf{F}$

Bitter neuron activation: fed vs. starved
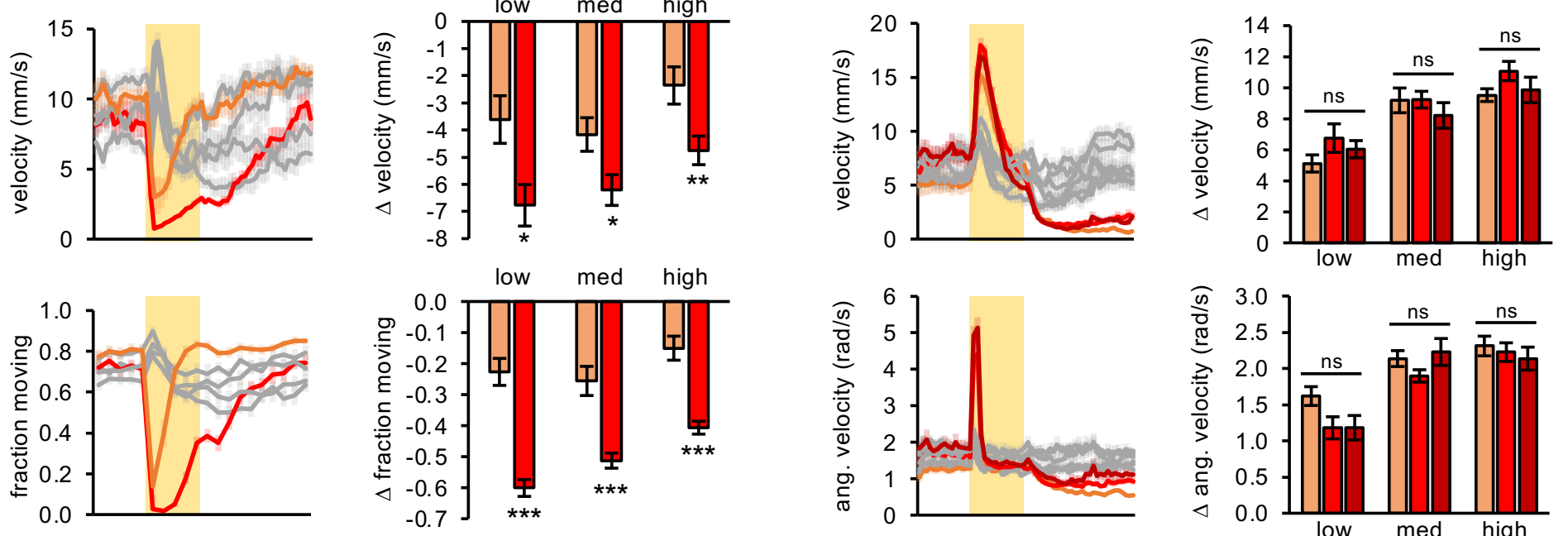

controls

Gr64f-Gal4/UAS-Chrim: fed vs. 1d starved
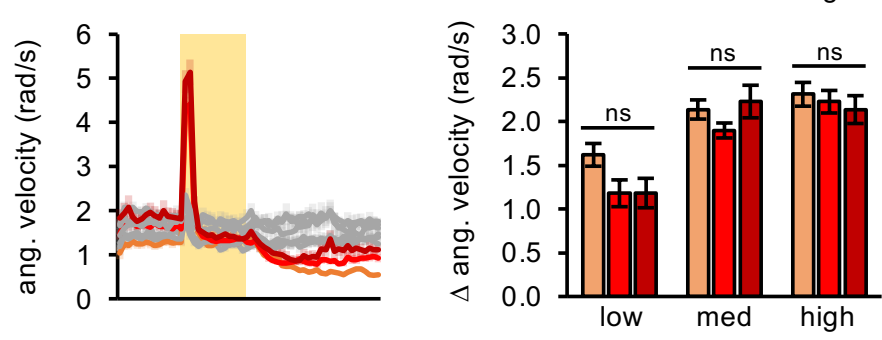

controls Gr33a-Gal4/UAS-Chrim: fed vs. 1d or 2d starved

Sugar + bitter neuron activation

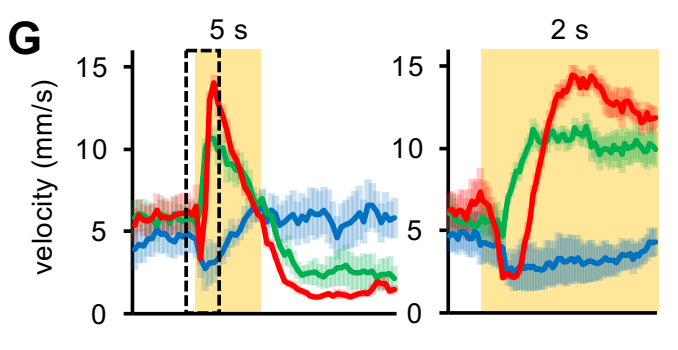

Gr64f-Gal4/UAS-Chrim

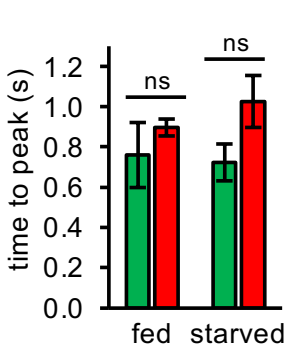

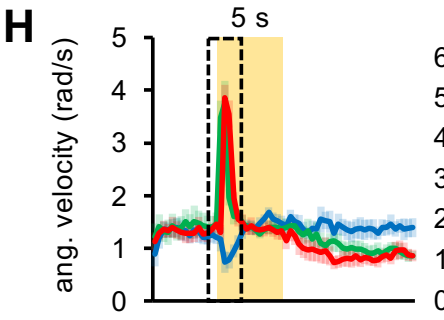
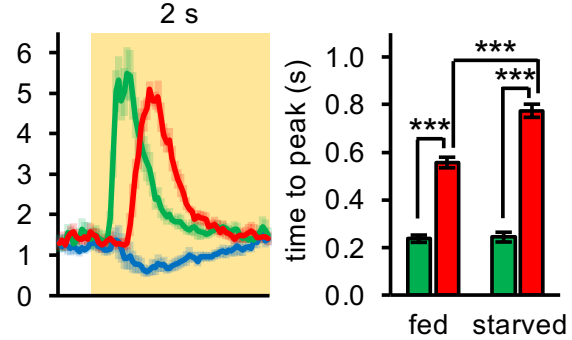
Figure 1. Activating sugar- or bitter-sensing neurons has opposing effects on locomotion. (A) Arena used for tracking flies during optogenetic activation. (B) Schematic of protocol to test the effect of taste neuron activation on locomotion. (C-F) Locomotor effects induced by activating sugar-sensing neurons using Gr64f-Gal4 (C) or bitter-sensing neurons using Gr33a-Gal4 (D) in fed flies ( $n=11-12$ trials). 5 sec light stimulation was used at 3 different intensities (low, medium, high). Left graphs show forward (top) and angular (bottom) velocity over time for medium light intensity (shading indicates light on). Bar graphs on the right quantify the change in these parameters at light onset (first $1 \mathrm{sec}$ ). (E-F) Effect of starvation on locomotor effects induced by activating sugar $(E)$ or bitter $(F)$ neurons ( $n=10-12$ trials). (E) Traces on left show forward velocity and fraction moving for low intensity light stimulation, for which the starvation effect is most apparent. Bar graphs quantify the change in these parameters averaged over the entire $5 \mathrm{sec}$ light stimulation; changes during the first $1 \mathrm{sec}$ of light were similar in fed and starved flies. ( $F$ ) Traces show forward and angular velocity for medium intensity light stimulation. (G-H) Effect of simultaneously activating sugar and bitter neurons using Gr64f-Gal4 + Gr33a-Gal4 on forward $(\mathrm{G})$ and angular $(\mathrm{H})$ velocity $(\mathrm{n}=9-10$ trials). Traces show behavior of fed flies with low intensity light stimulation. Right traces show zoomed in behavior around light onset (boxes shown in left graphs). Bar graphs quantify the time to peak velocity following light onset for fed versus one-day starved flies when bitter neurons were activated alone (green) or along with sugar neurons (red). Gal4/+ and UAS/+ controls are not shown here but were tested alongside the experimentals and behaved similarly to controls shown in panels $C-F$.

For all figures: ${ }^{*} p<0.05,{ }^{* *} p<0.01,{ }^{* * *} p<0.001$, ns $=$ not significant $(p>0.05)$. Two groups were compared using unpaired t-tests and more than two groups were compared using one-way ANOVA followed by Dunnett's test comparing experimental flies to each control. 

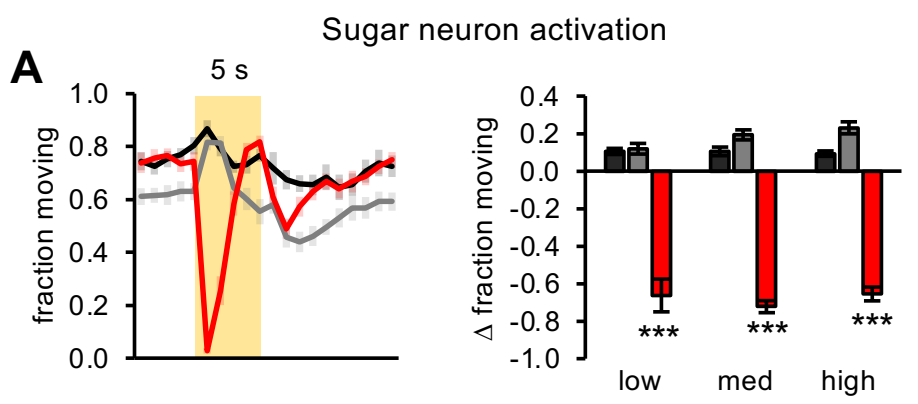

Gr64f-Gal4/+ UAS-Chrim/+ Gr64f-Gal4/UAS-Chrim
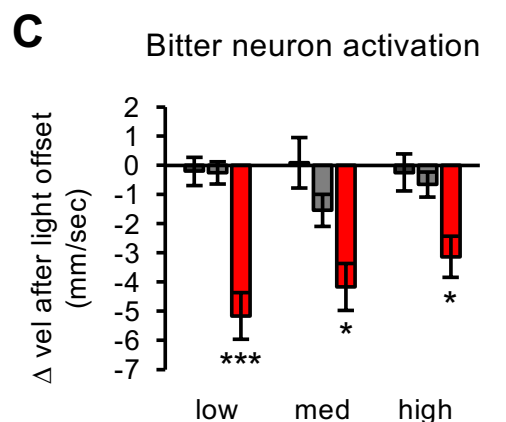

Gr33a-Gal4/+ UAS-Chrim/+
B
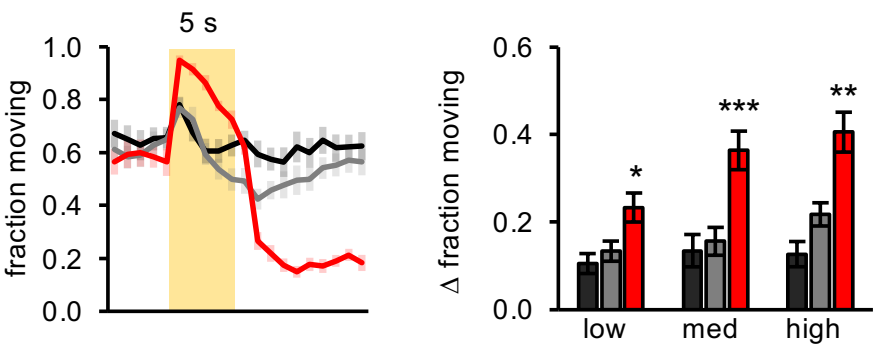

Gr33a-Gal4/+ UAS-Chrim/+

Gr33a-Gal4/UAS-Chrim

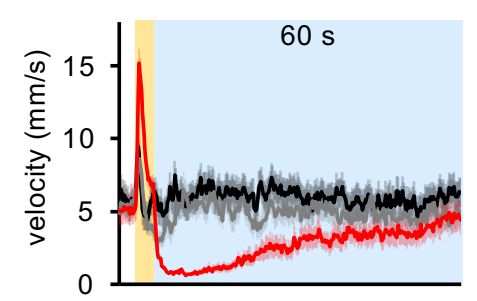

Gr33a-Gal4/UAS-Chrim

Figure 1 - Figure Supplement 1. Further characterization of locomotor effects induced by sugar or bitter neuron activation. (A-B) Fraction of flies moving when sugar (A) or bitter (B) neurons were activated ( $n=11-12$ trials). Traces show behavior with medium intensity light stimulation. Bar graphs show change in behavior at light onset. (C) Locomotor suppression following bitter activation ( $n=11-12$ trials). (D) Post-light locomotor suppression persists for nearly a minute. Graphs shows velocity during the first $60 \mathrm{sec}$ (blue shading) after $5 \mathrm{sec}$ stimulation with medium intensity light (yellow shading); same experiment as shown in Figure 1D ( $n=11-12$ trials). 


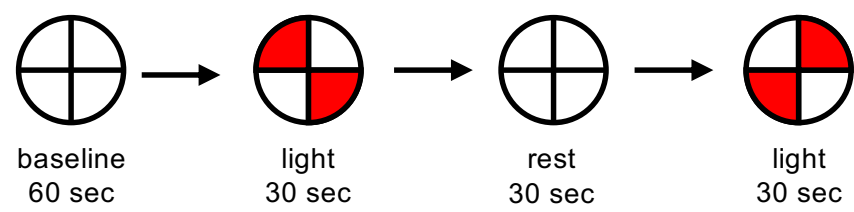

Sugar neuron activation

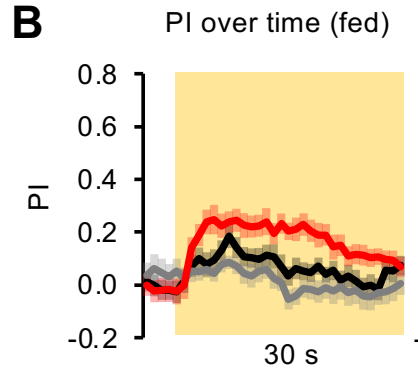

Gr64f-Gal4/+ UAS-Chrim/+

PI over time (starved)

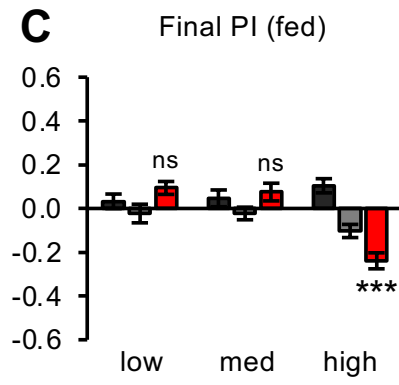

Gr64f-Gal4/UAS-Chrim

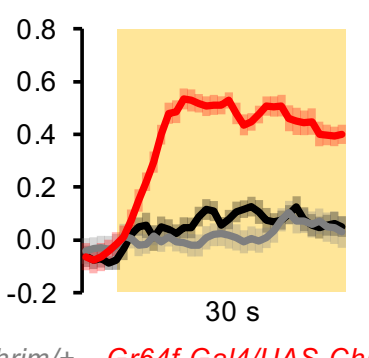

E

PI over time (fed)

PI over time (starved)
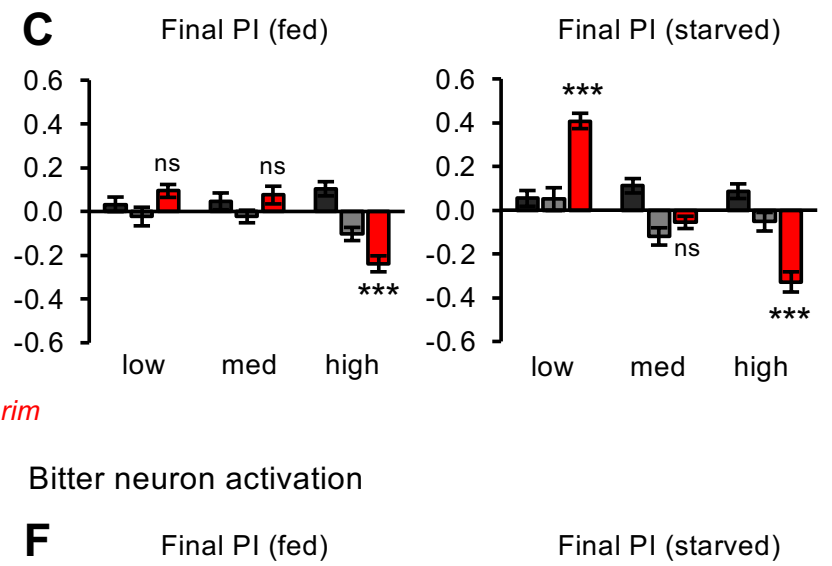

D Fed vs. starved
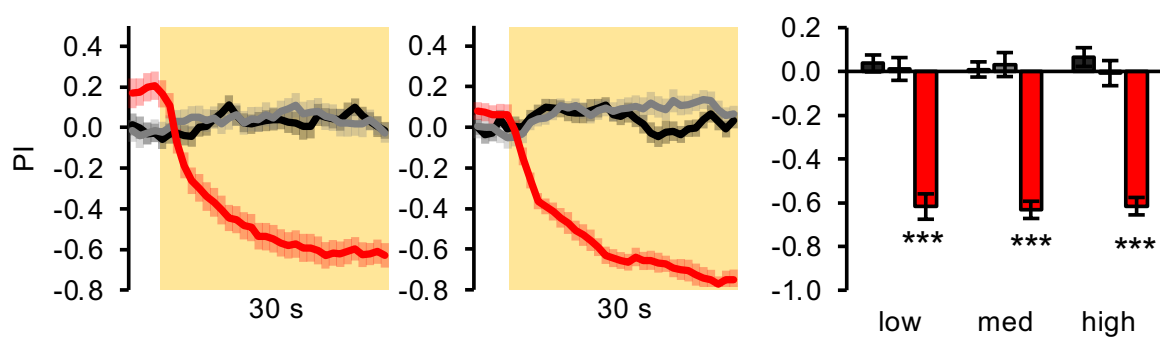

Gr33a-Gal4/+ UAS-Chrim/+ Gr33a-Gal4/UAS-Chrim

Sugar + bitter neuron activation

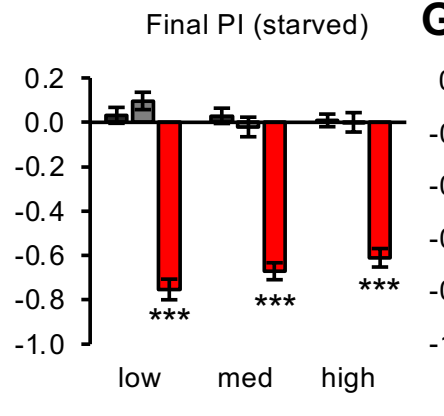

H Pl over time (starved)

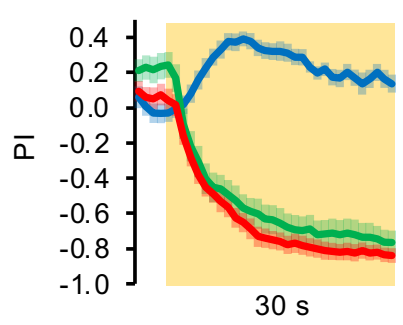

Gr64f-Gal4/ UAS-Chrim
I

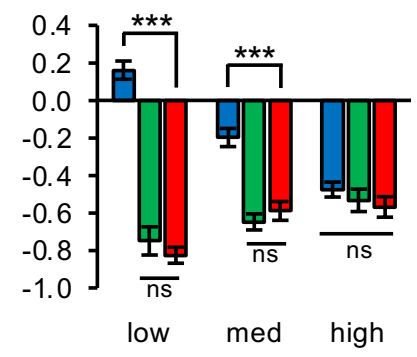

Gr64f-Gal4, Gr33a-Gal4/ UAS-Chrim
J Fed vs. starved

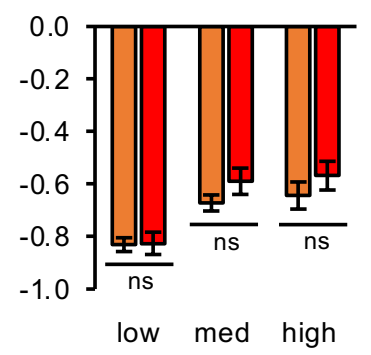

fed 1d starved

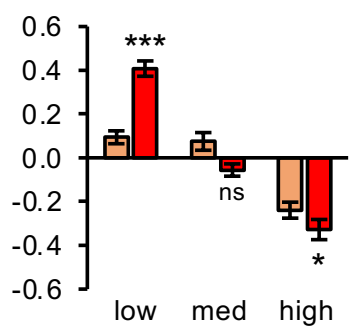

fed $1 d$ starved

G Fed vs. starved

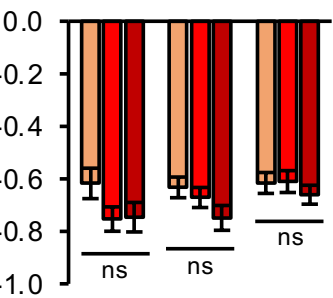

low med high

fed $1 \mathrm{~d}$ starved 2d starved

Figure 2. Activating sugar- or bitter-sensing neurons has opposing effects on positional preference. (A) Schematic of protocol to test the effect of taste neuron activation on innate positional preference (adapted from Aso et al., 2014). (B-J) Effect of activating sugar-sensing (B-D), bitter-sensing (E-G), or both sugar- and bitter-sensing neurons $(\mathrm{H}-\mathrm{J})$ on positional preference $(n=$ 18-24 trials, 9-12 sets of flies). Light preference is quantified by the preference index (PI): (\# flies in light quadrants - \# flies in nonlight quadrants) / total \# flies. Line graphs in panels $B, E$, and $\mathrm{H}$ show PI over 30 sec of low intensity light presentation (shading indicates light on), including both of the sequential test periods when the lighted quadrants were switched (A). Flies with bitter activation sometimes appear to show a positive $\mathrm{PI}$ at baseline because of the repeated tests: after the first test they continued to avoid the previously illuminated quadrants until the next test. Preference traces for other light intensities are shown in Figure 2 Figure Supplement 1. Panels C, F, and I show the final PI (average over last 5 sec of light presentation) for control vs. experimental flies. Panels D, G, and $\mathrm{J}$ show the final PI of fed vs. starved experimental flies. For panel $\mathrm{J}$ the experimental flies are flies in which both sugar and bitter neurons are activated. Gal4/+ and UAS/+ controls are not shown in panels H-J but were tested alongside the experimentals and behaved similarly to controls shown in panels B-G. 
B Sugar neuron activation: 1d starved
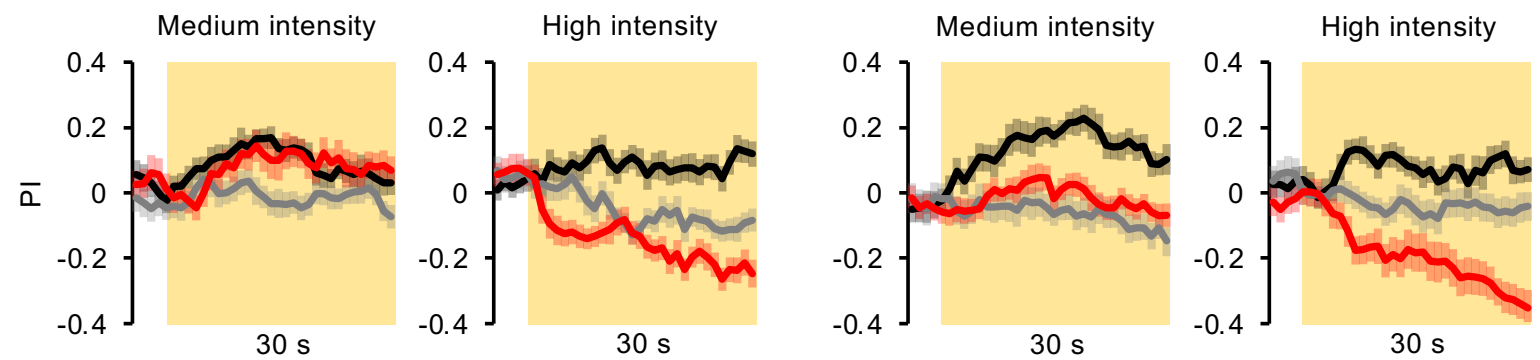

Gr64f-Gal4/+ UAS-Chrim/+ Gr64f-Gal4/UAS-Chrim

C

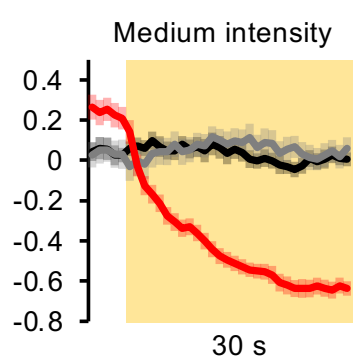

Bitter neuron activation: fed

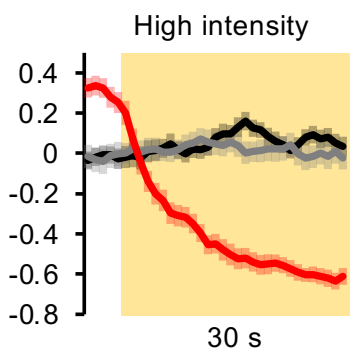

D

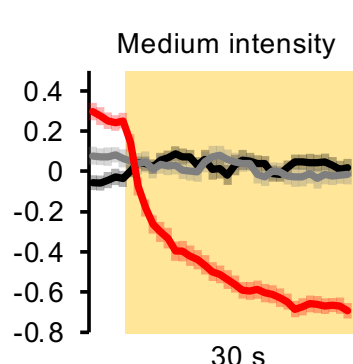

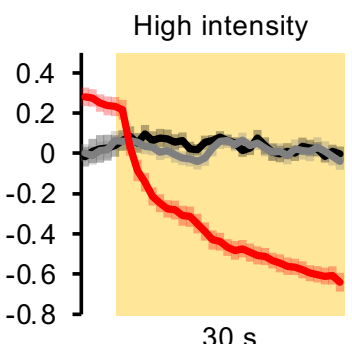

$30 \mathrm{~s}$

Gr33a-Gal4/+ UAS-Chrim/+ Gr33a-Gal4/UAS-Chrim

Figure 2 - Figure Supplement 1. Positional preference at other light intensities. Effect of activating sugar neurons (A$B)$ or bitter neurons (C-D) at medium or high light intensity in fed (A, C) or one-day starved (B, D) flies $(n=22-24$ trials, 1112 sets of flies). Traces for low light intensity and statistical analysis of final PI values are shown in Figure 2. 
A

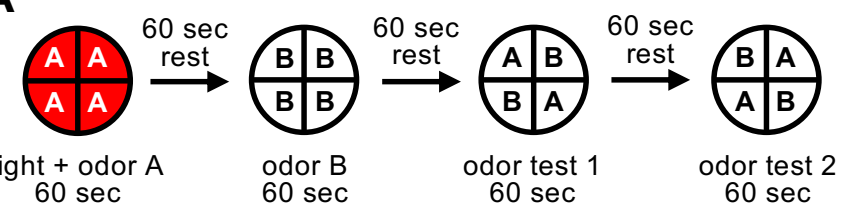

Sugar neuron activation

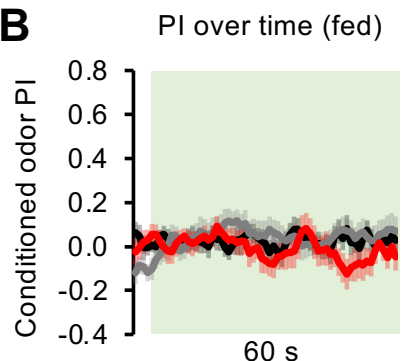

Gr64f-Gal4/+ UAS-Chrim/+
Pl over time (starved)

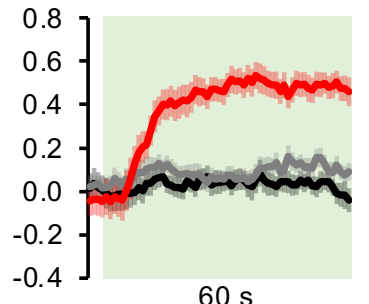

Gr64f-Gal4/UAS-Chrim
C

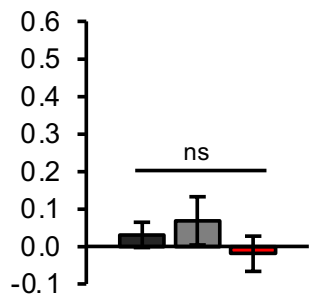

Final PI (starved)

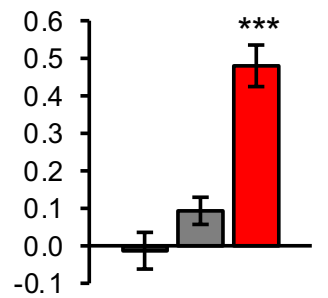

D Fed vs. starved

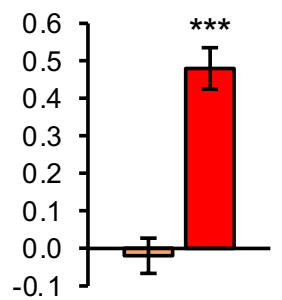

fed

$1 \mathrm{~d}$ starved

Bitter neuron activation

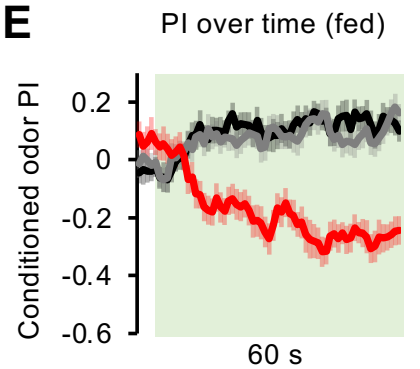

Gr33a-Gal4/+
PI over time (starved)

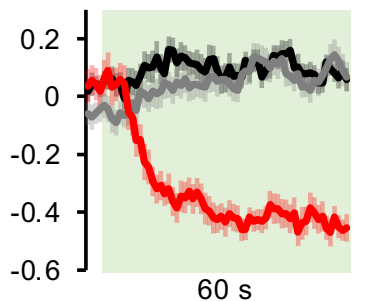

Gr33a-Gal4/UAS-Chrim
F Final PI (fed)

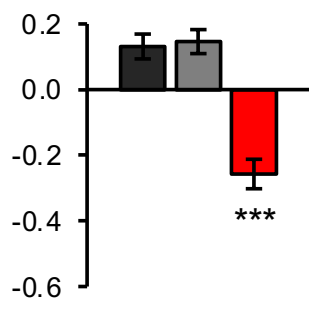

Final PI (starved)

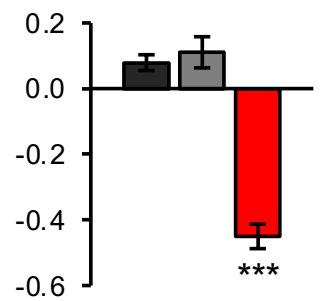

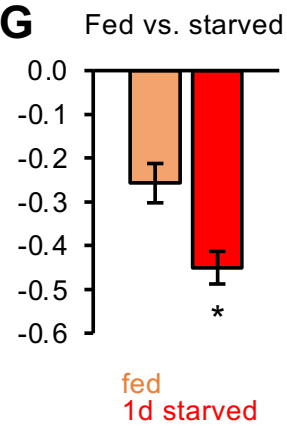

Sugar + bitter neuron activation
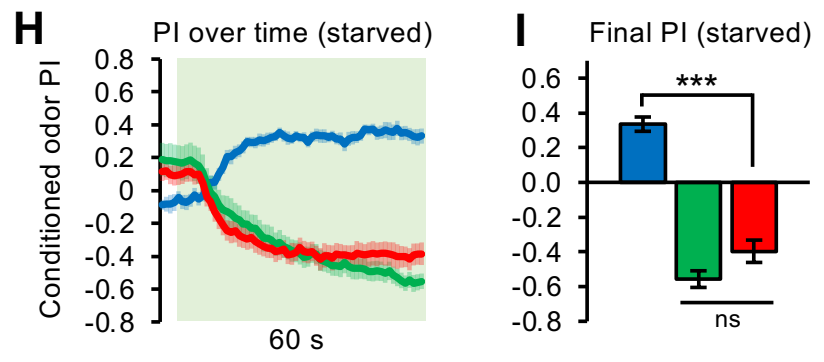

$\begin{array}{lcc}\text { Gr64f-Gal4/ } & \text { Gr33a-Gal4/ } & \text { Gr64f-Gal4, Gr33a-Gal4/ } \\ \text { UAS-Chrim } & \text { UAS-Chrim } & \text { UAS-Chrim }\end{array}$

Figure 3. Activating sugar- or bitter-sensing neurons has opposing effects on learned odor preference. (A) Schematic of protocol to test the effect of taste neuron activation on learned odor preference (adapted from Aso \& Rubin, 2016). Learned preference was quantified as the PI for the conditioned odor during the test periods, calculated as: (\# flies in CS+ quadrants - \# flies in CS- quadrants) / total \# flies. Data from test 1 and 2 are combined in all figures. (B-G) Effects of activating sugar-sensing neurons (B-D) or bitter-sensing neurons (E-G) on learned preference in fed and 1d starved flies ( $n=16-24$ trials, 8-12 sets of flies). (H-I) Effect of simultaneously activating sugar- and bitter-sensing neurons in $1 \mathrm{~d}$ starved flies ( $\mathrm{n}=20$ trials, 10 sets of flies). Gal4/+ and UAS/+ controls are not shown here but were tested alongside the experimentals and behaved similarly to controls shown in panels B-G. Line graphs in panels $\mathrm{B}, \mathrm{E}$, and $\mathrm{H}$ show the conditioned odor PI over time during the odor test periods (shading indicates odors on). Test 1 and test 2 were combined. Flies with bitter activation sometimes appear to show a positive PI at baseline because of the repeated tests: after test 1 they continued to avoid the previous CS+ quadrants until test 2 when the odor quadrants were switched. Panels C, F, and I show the final PI for the conditioned odor (average over last 5 sec of test) for different genotypes. Panels D and G show final PI of fed vs. starved experimental flies. 


\begin{tabular}{|c|c|c|c|c|c|}
\hline & $\begin{array}{c}\text { Sugar } \\
\text { neurons } \\
\text { (fed) }\end{array}$ & $\begin{array}{c}\text { Sugar } \\
\text { neurons } \\
\text { (1d starved) }\end{array}$ & $\begin{array}{c}\text { Bitter } \\
\text { neurons } \\
(\text { fed })\end{array}$ & $\begin{array}{c}\text { Bitter } \\
\text { neurons } \\
\text { (1d starved) }\end{array}$ & $\begin{array}{c}\text { Bitter } \\
\text { neurons } \\
(2 d \text { starved })\end{array}$ \\
\hline $\begin{array}{l}\text { Light-induced } \\
\text { locomotion }\end{array}$ & $\begin{array}{l}\text { moderate } \\
\text { decrease }\end{array}$ & $\begin{array}{c}\text { strong } \\
\text { decrease }\end{array}$ & $\begin{array}{l}\text { moderate } \\
\text { increase }\end{array}$ & $\begin{array}{l}\text { moderate } \\
\text { increase }\end{array}$ & $\begin{array}{c}\text { strong } \\
\text { increase }\end{array}$ \\
\hline $\begin{array}{l}\text { Light-induced } \\
\text { turning }\end{array}$ & $\begin{array}{c}\text { strong } \\
\text { decrease }\end{array}$ & $\begin{array}{c}\text { strong } \\
\text { decrease }\end{array}$ & $\begin{array}{c}\text { strong } \\
\text { increase }\end{array}$ & $\begin{array}{c}\text { strong } \\
\text { increase }\end{array}$ & $\begin{array}{c}\text { strong } \\
\text { increase }\end{array}$ \\
\hline $\begin{array}{l}\text { Post-light } \\
\text { locomotion }\end{array}$ & not analyzed & not analyzed & $\begin{array}{l}\text { moderate } \\
\text { decrease }\end{array}$ & $\begin{array}{c}\text { strong } \\
\text { decrease }\end{array}$ & $\begin{array}{c}\text { strong } \\
\text { decrease }\end{array}$ \\
\hline $\begin{array}{c}\text { Innate } \\
\text { preference }\end{array}$ & no effect & $\begin{array}{l}\text { strong } \\
\text { attraction }\end{array}$ & $\begin{array}{l}\text { strong } \\
\text { aversion }\end{array}$ & $\begin{array}{l}\text { strong } \\
\text { aversion }\end{array}$ & $\begin{array}{l}\text { strong } \\
\text { aversion }\end{array}$ \\
\hline $\begin{array}{l}\text { Learned } \\
\text { preference }\end{array}$ & no effect & $\begin{array}{l}\text { strong } \\
\text { attraction }\end{array}$ & $\begin{array}{c}\text { moderate } \\
\text { aversion }\end{array}$ & $\begin{array}{l}\text { strong } \\
\text { aversion }\end{array}$ & not tested \\
\hline
\end{tabular}

B Integrative model

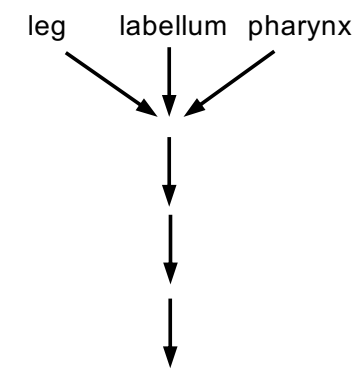

Common behavioral effects

\section{Segregated processing model}

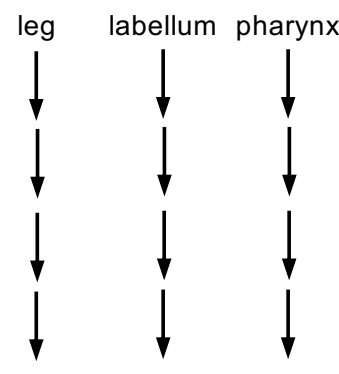

Distinct behavioral effects

Figure 4. Summary of behavioral effects and models for taste processing. (A) Summary of behavioral effects of sugar or bitter neuron activation. Effects are color-coded by direction and strength; see Materials and Methods for explanation of how effects were quantified. For sugar-sensing neurons, only the attractive innate preference induced by low light intensities was analyzed. (B) Two models for how different taste inputs within a single taste modality (e.g. inputs from different organs) may be processed downstream to regulate behavior. 
Locomotor changes

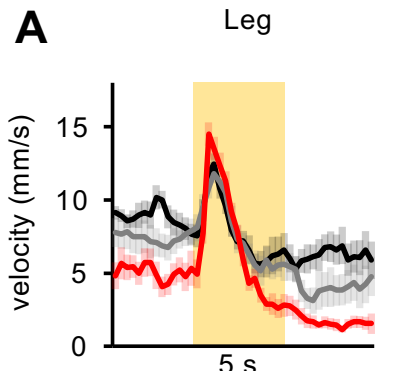

Labellum

Labellum (S-b)

Pharynx
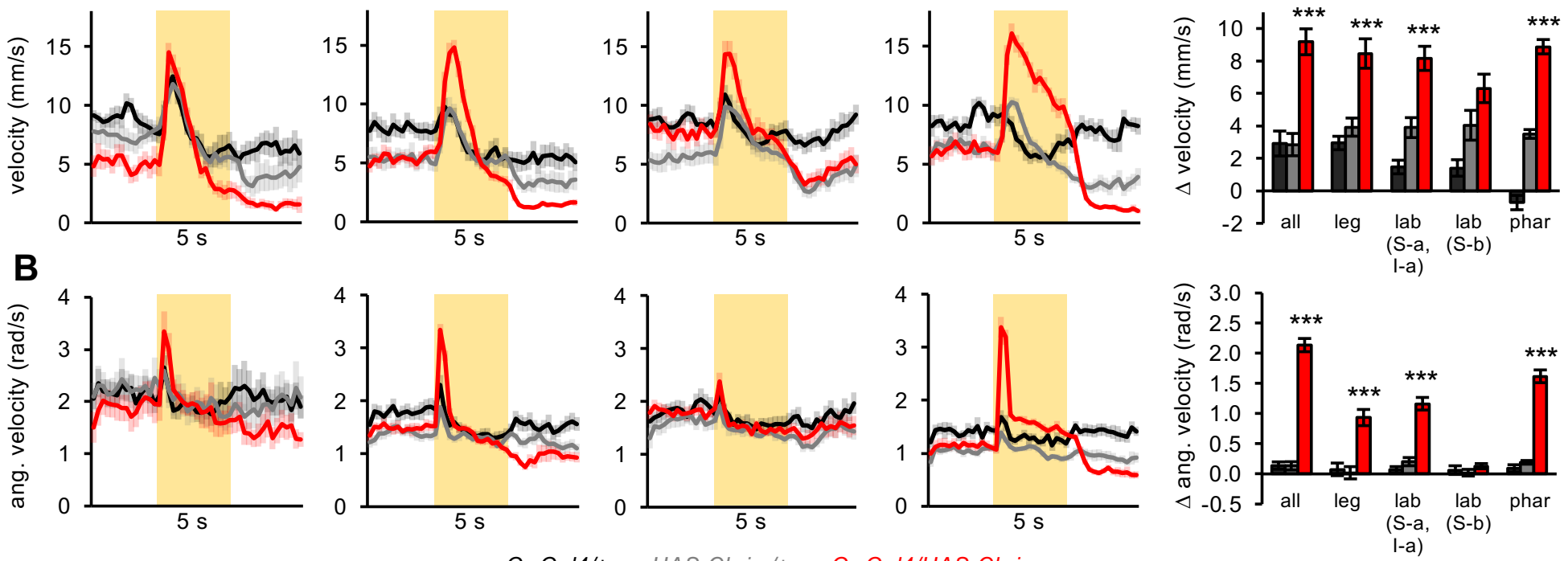

Innate preference
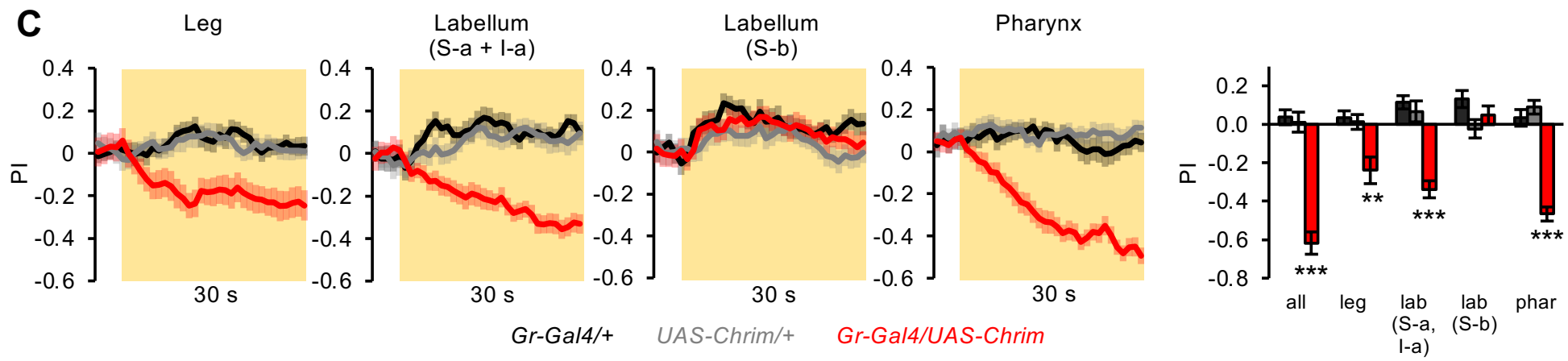

Learned odor preference
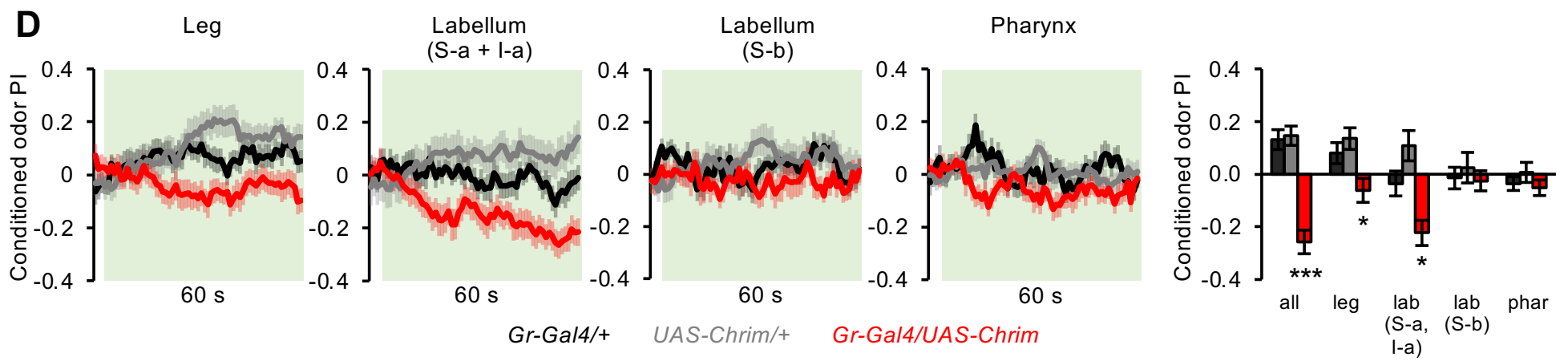

Suppression of PER
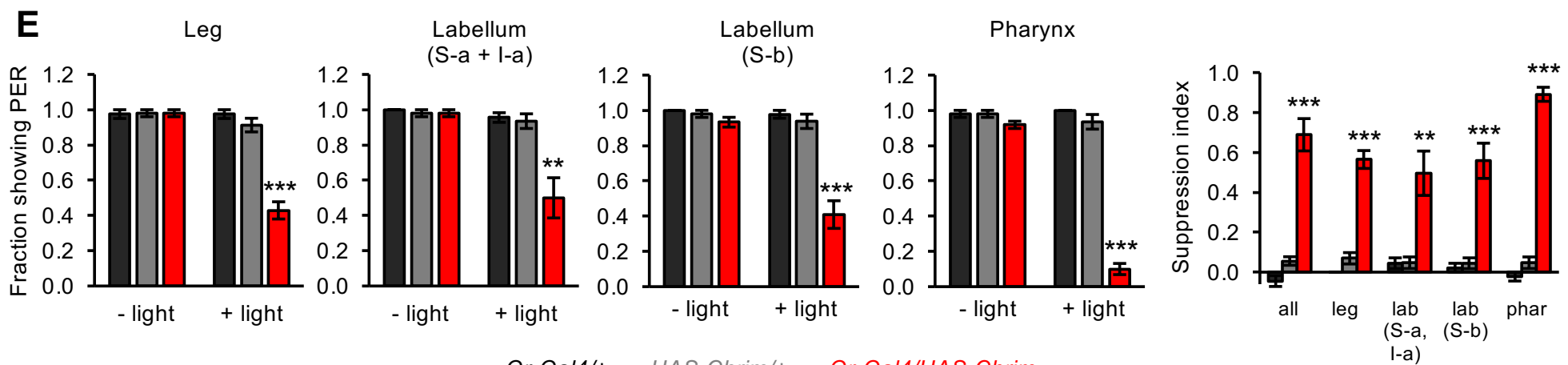
Figure 5. Activating subsets of bitter-sensing neurons affects locomotion, innate and learned preference, and feeding responses. (A-B) Locomotor effects induced by activating bitter neuron subsets with medium intensity light ( $n=10-12$ trials). Effects of other light intensities are shown in Figure 5 - Figure Supplement 1. Traces show forward $(A)$ or angular $(B)$ velocity before, during, and after $5 \mathrm{sec}$ light presentation (shaded). Bar graphs quantify the change in these parameters at light onset. (C) Effect of activating bitter neuron subsets with low intensity light on innate avoidance ( $\mathrm{n}=20-24$ trials, 10-12 sets of flies). Effects at other light intensities are shown in Figure 5 - Figure Supplement 2. Traces show PI over 30 sec light presentation (shaded), and bar graph shows PI during last $5 \mathrm{sec}$ of light presentation. (D) Effect of activating bitter neuron subsets on learned odor preference $(n=20-24$ trials, $10-12$ sets of flies). Traces show PI for conditioned odor over 60 sec of odor presentation (shaded) after learning (test 1 and 2 combined). Bar graph shows PI during last $5 \mathrm{sec}$ of odor presentation. (E) Effect of activating bitter neuron subsets on PER to $100 \mathrm{mM}$ sucrose ( $\mathrm{n}=5-8$ sets of flies). Bar graphs for each neuronal subset (left) represent the fraction of flies showing PER with and without light stimulation. Bar graph on the right shows the degree of PER suppression elicited by neuronal activation, quantified as: 1 - (PER with light / PER without light).

In all panels, Gal4 lines used were Gr58c-Gal4 (leg), Gr59c-Gal4 (labellum, S-a + I-a), Gr22f-Gal4 (labellum, S-b), Gr9a-Gal4 (pharynx), and Gr33a-Gal4 (all bitter neurons). Experimental effects not labeled with an asterisk are not significant. 
A

Low intensity

High intensity
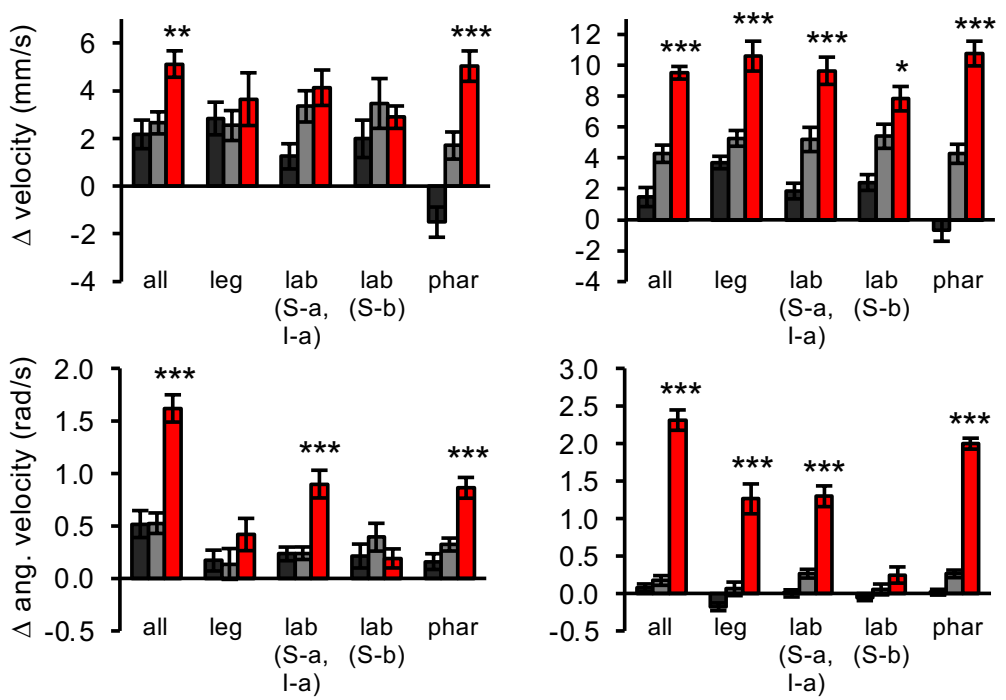

Gr-Gal4/+ UAS-Chrim/+ Gr-Gal4/UAS-Chrim
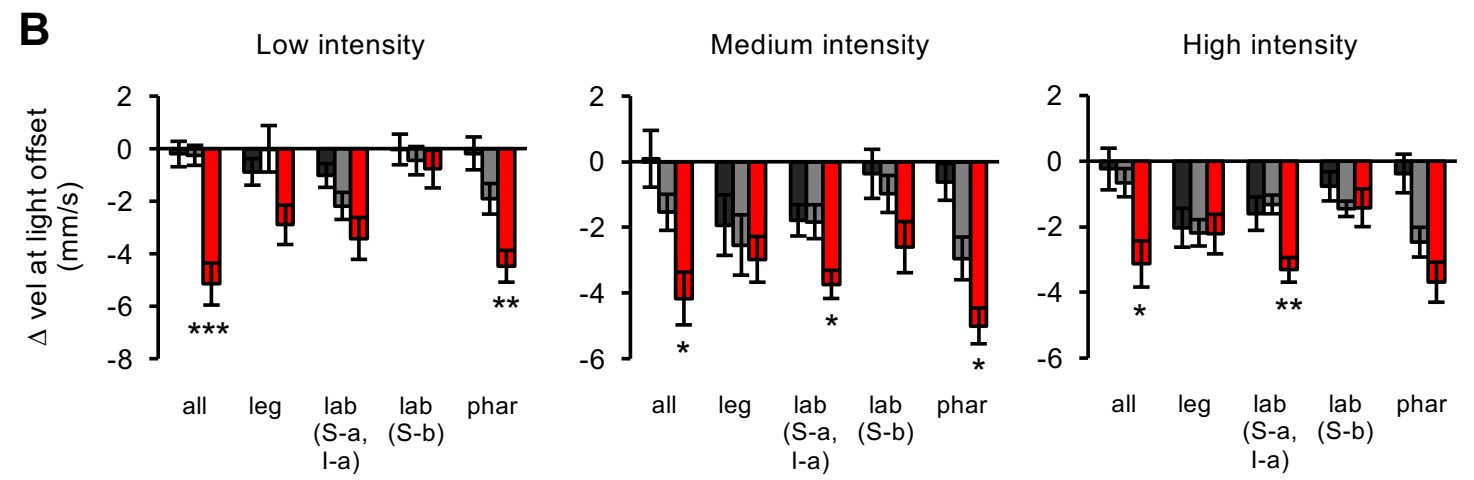

Gr-Gal4/+

UAS-Chrim/+

Gr-Gal4/UAS-Chrim

Figure 5 - Figure Supplement 1. Additional characterization of locomotor effects elicited by activating subsets of bitter neurons. (A) Change in forward velocity (top) or angular velocity (bottom) induced by low or high intensity light stimulation $(\mathrm{n}=$ 10-12 trials). Results for medium intensity stimulation are shown in Figure 5. (B) Quantification of locomotor suppression elicited by the termination of optogenetic stimulation at low, medium, or high light intensity ( $n=10-12$ trials).

For all graphs, experimental effects not labeled with an asterisk are not significant. Gal4 lines used were Gr58c-Gal4 (leg), Gr59c-Gal4 (labellum, S-a + I-a), Gr22f-Gal4 (labellum, S-b), Gr9a-Gal4 (pharynx), and Gr33a-Gal4 (all bitter neurons). 
A

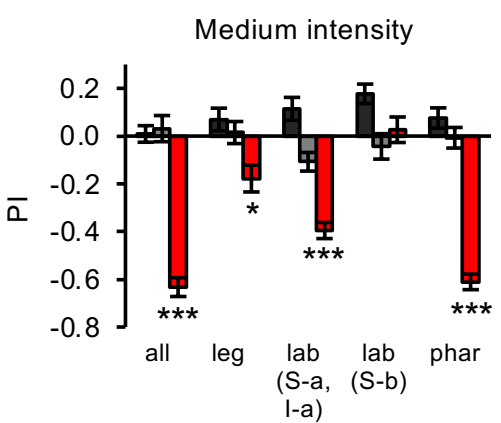

Gr-Gal4/+ $\quad$ UAS-Chrim/+
B High intensity

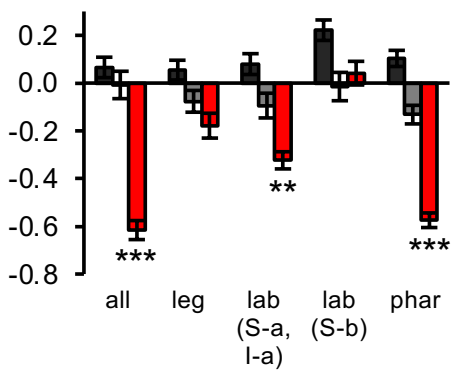

Gr-Gal4/UAS-Chrim

Figure 5 - Figure Supplement 2. Innate positional preference elicited by activating bitter neuron subsets at other light intensities. PI during last $5 \mathrm{sec}$ of light presentation at medium (A) or high (B) light intensity ( $n=20-24$ trials, 10-12 sets of flies). Effect of low intensity light is shown in Figure 5. Experimental effects not labeled with an asterisk are not significant. Gal4 lines used were Gr58c-Gal4 (leg), Gr59c-Gal4 (labellum, S-a + I-a), Gr22f-Gal4 (labellum, S-b), Gr9a-Gal4 (pharynx), and Gr33a-Gal4 (all bitter neurons). 


\begin{tabular}{|c|c|c|c|c|c|}
\hline & $\begin{array}{c}\text { All bitter } \\
\text { neurons }\end{array}$ & Leg & $\begin{array}{c}\text { Labellum } \\
\mathbf{( S - a}+\mathbf{I - a})\end{array}$ & $\begin{array}{c}\text { Labellum } \\
\mathbf{( S - b )}\end{array}$ & Pharynx \\
\hline $\begin{array}{c}\text { Light-induced } \\
\text { locomotion }\end{array}$ & $\begin{array}{c}\text { strong } \\
\text { increase }\end{array}$ & $\begin{array}{c}\text { strong } \\
\text { increase }\end{array}$ & $\begin{array}{c}\text { moderate } \\
\text { increase }\end{array}$ & $\begin{array}{c}\text { weak } \\
\text { increase }\end{array}$ & $\begin{array}{c}\text { strong } \\
\text { increase }\end{array}$ \\
\hline $\begin{array}{c}\text { Light-induced } \\
\text { turning }\end{array}$ & $\begin{array}{c}\text { strong } \\
\text { increase }\end{array}$ & $\begin{array}{c}\text { moderate } \\
\text { increase }\end{array}$ & $\begin{array}{c}\text { moderate } \\
\text { increase }\end{array}$ & no effect & $\begin{array}{c}\text { strong } \\
\text { increase }\end{array}$ \\
\hline $\begin{array}{c}\text { Post-light } \\
\text { locomotion }\end{array}$ & $\begin{array}{c}\text { strong } \\
\text { decrease }\end{array}$ & no effect & $\begin{array}{c}\text { weak } \\
\text { decrease }\end{array}$ & no effect & $\begin{array}{c}\text { moderate } \\
\text { decrease }\end{array}$ \\
\hline $\begin{array}{c}\text { Innate } \\
\text { preference }\end{array}$ & $\begin{array}{c}\text { strong } \\
\text { aversion }\end{array}$ & $\begin{array}{c}\text { weak } \\
\text { aversion }\end{array}$ & $\begin{array}{c}\text { moderate } \\
\text { aversion }\end{array}$ & no effect & $\begin{array}{c}\text { strong } \\
\text { aversion }\end{array}$ \\
\hline $\begin{array}{c}\text { Learned } \\
\text { preference }\end{array}$ & $\begin{array}{c}\text { strong } \\
\text { aversion }\end{array}$ & $\begin{array}{c}\text { weak } \\
\text { aversion }\end{array}$ & $\begin{array}{c}\text { moderate } \\
\text { aversion }\end{array}$ & no effect & no effect \\
\hline $\begin{array}{c}\text { Suppression } \\
\text { of PER }\end{array}$ & $\begin{array}{c}\text { strong } \\
\text { suppression }\end{array}$ & suppression & $\begin{array}{c}\text { moderate } \\
\text { suppression }\end{array}$ & suppression & suppression \\
\hline
\end{tabular}

Figure 6. Summary of behavioral effects induced by activating bitter neuron subsets. Effects are color-coded by strength, relative to the effect of activating all bitter neurons. All observed effects went in the same direction. See Materials and Methods for details of how effects were quantified. 
A All bitter neurons

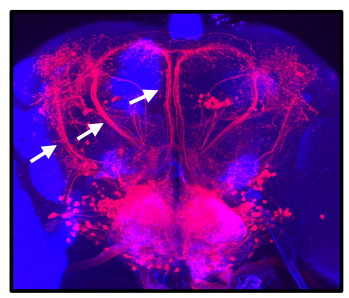

B

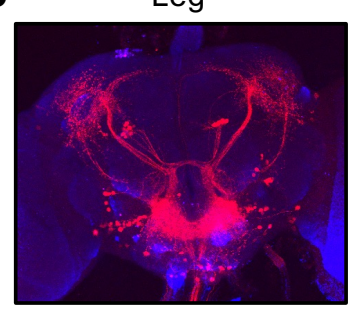

Labellum $(\mathrm{S}-\mathrm{a}+\mathrm{I}-\mathrm{a})$

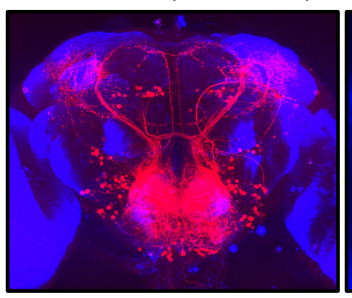

Pharynx

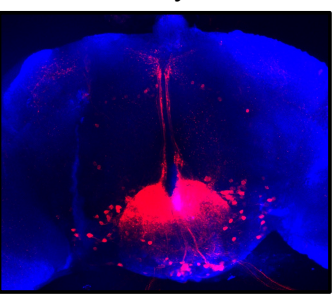

C leg labellum pharynx

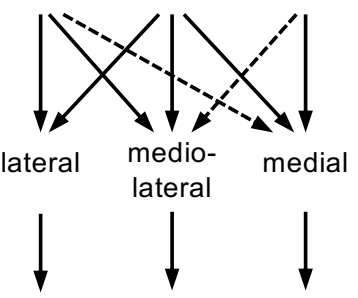

Figure 7. Bitter inputs from different organs are relayed to overlapping downstream regions. (A) The entire population of second-order bitter neurons was labeled by trans-Tango tracing from Gr33a-Gal4-expressing cells. Arrows denote the three major tracts (lateral, mediolateral, and medial) projecting out of the SEZ to the dorsal brain. (B) Neurons postsynaptic to specific bitter neuron subsets were labeled with trans-Tango. Gal4 lines used were Gr58c-Gal4 (leg), Gr59c-Gal4 (labellar S-a + I-a), and Gr9aGal4 (pharynx). Tracing from labellar S-b cells labeled by Gr22f-Gal4 yielded very weak trans-Tango staining that was not conclusive, so is not shown here. (C) Summary of inputs from different subsets of bitter neurons onto second-order projection pathways. Dotted lines indicate weak input based on weak or inconsistent trans-Tango staining. 
A
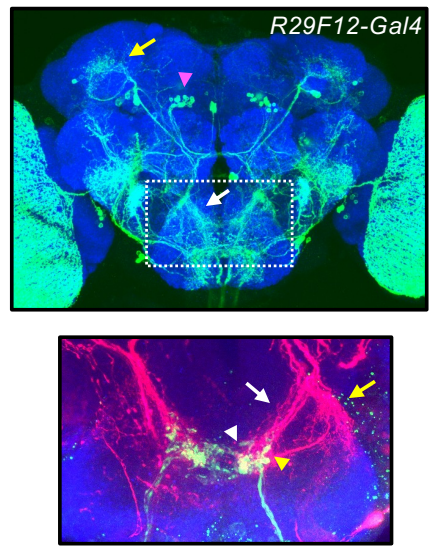

Gr66a-lexA > lexAop-GCaMP6f R29F12-Gal4 > UAS-TdTomato

D

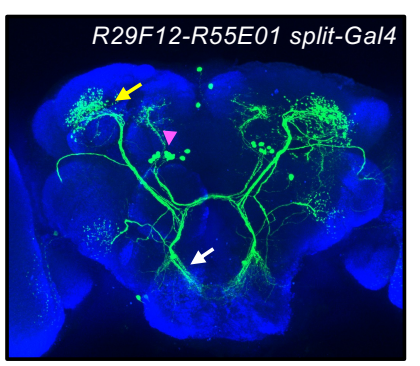

B

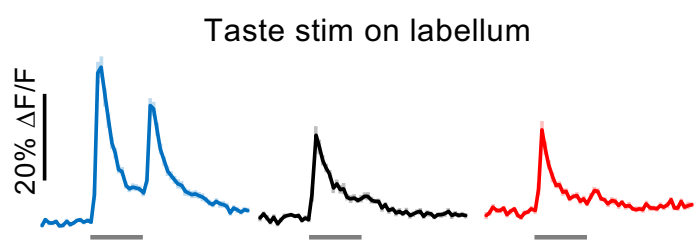

C

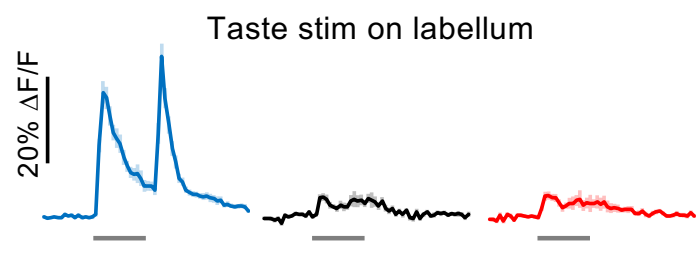

Taste stim on leg

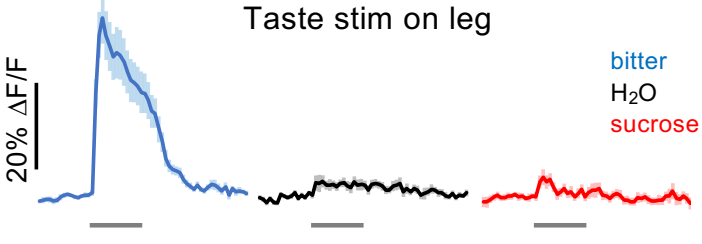

SLP responses

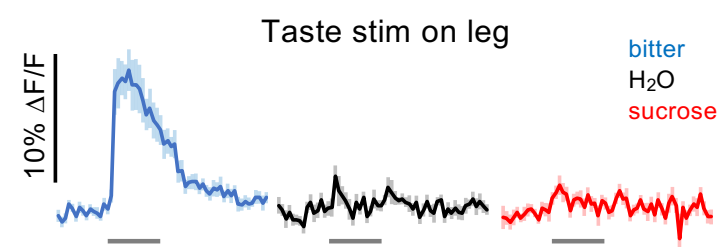

E Neuronal activation: F

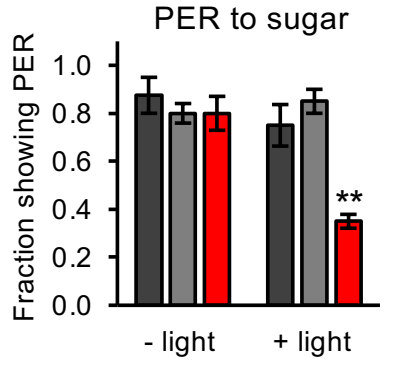

split-Gal4/+ UAS-Chrim/+ split-Gal4/UAS-Chrim

Forward speed

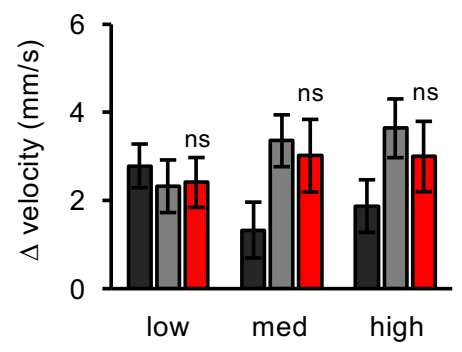

split-Gal4/+ UAS-Chrim/+
Neuronal silencing: PER to sugar +/- bitter

F

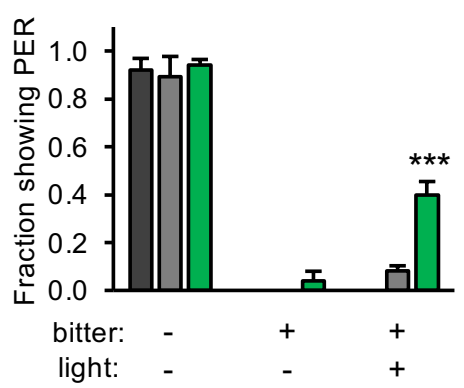

split-Gal4/+ UAS-Chrim/+

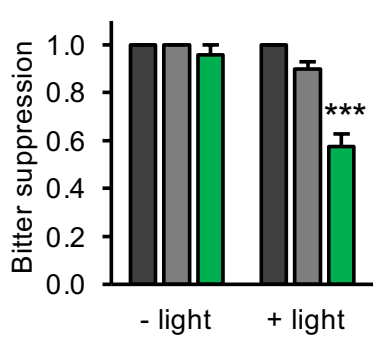

split-Gal4/UAS-GTACR1
Turning
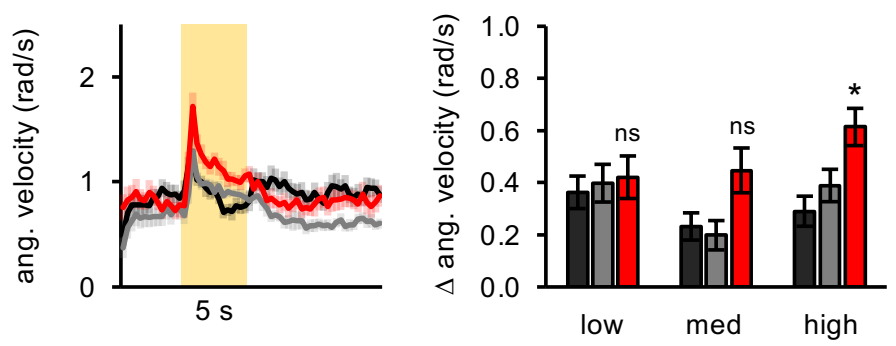

split-Gal4/UAS-Chrim ter crose crose
G

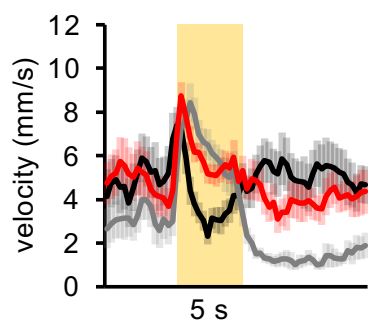

H

Innate preference

Final PI
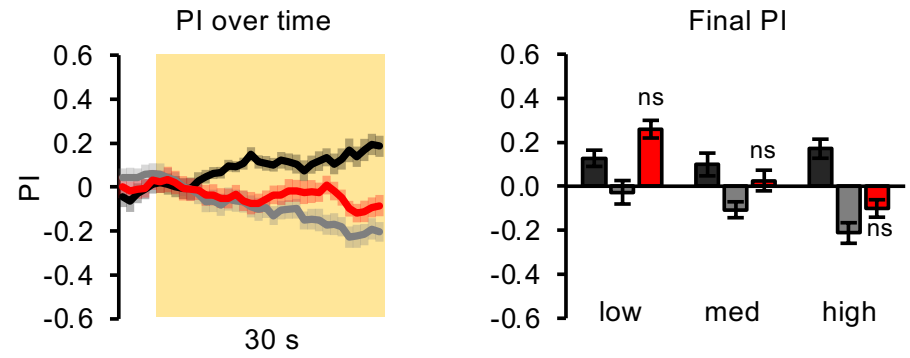

split-Gal4/+ UAS-Chrim/+ 
Figure 8: Second-order mISEZt bitter neurons receive input from multiple organs and regulate a subset of behaviors.

(A) Top: R29F12-Gal4 expression pattern (maximum intensity projection created from images generated by the Janelia FlyLight Project Team). Brain slices at the far anterior and posterior edges are not included to maximize visibility of mISEZt cells. Pink arrowhead shows mISEZt cell bodies; white and yellow arrows show mISEZt projections in the SEZ and SLP, respectively. Box depicts approximate area of the SEZ shown in the bottom image. Bottom: SEZ image with mISEZt neurons (R29F12-Gal4 driving UAS-tdTomato, red) colabeled with bitter-sensing neurons (Gr66a-lexA driving lexAop-GCaMP6f, green). Medial and lateral branches of the mISEZt projections are denoted by white and yellow arrows, respectively. Based on morphology, most of the visible bitter sensory projections likely arise from the labellum (white arrowhead); bitter axons from the leg have a sticklike projection just lateral to the labellar projections (yellow arrowhead). (B-C) GCaMP responses of mISEZt projections in the SEZ (B) or SLP (C) with taste stimuli applied to the labellum (left) or leg (right). R29F12-Gal4 was used to drive UASGCaMP6f. For all imaging traces, the grey bar denotes $5 \mathrm{sec}$ taste presentation. The bitter stimulus was 10 mM denatonium. Different sets of flies were used for different experiments, so response magnitudes across different conditions are not directly comparable. (B) $n=15-22$ trials, 4 flies (labellar stimulation); $n=15-19$ trials, 4 flies (leg stimulation). (C) $n=25-34$ trials, 7 flies (labellar stimulation); $\mathrm{n}=12-19$ trials, 3-4 flies (leg stimulation). (D) Expression pattern of split-Gal4 line (R29F12-AD + R55E01-DBD) labeling mISEZt neurons with high specificity. Pink arrowhead shows mISEZt cell bodies; white and yellow arrows denote their projections in the SEZ and SLP, respectively. (E) Effect of activating mISEZt neurons on PER to $50 \mathrm{mM}$ sucrose ( $n=4$ experiments, 40 flies). (F) Effect of silencing mISEZt neurons on bitter suppression of PER, tested by adding 10 $\mathrm{mM}$ quinine to $50 \mathrm{mM}$ sucrose ( $\mathrm{n}=5$ experiments, $46-50$ flies). Left graph shows PER under each condition (3 of the 9 conditions produced zero PER, so bars are at zero with zero error). Right graph shows the degree of bitter suppression based on the left graph, quantified as: 1 - (PER with bitter / PER without bitter). (G-I) Effects of mISEZt neuron activation on locomotion ( $\mathrm{G} ; \mathrm{n}=11-12$ trials), innate preference $(\mathrm{H} ; \mathrm{n}=22-24$ trials, 11-12 sets of flies), and learned odor preference $(\mathrm{I} ; \mathrm{n}=$ 14-16 trials, 7-8 sets of flies). Effects of high intensity light stimulation are shown here; for other intensities see Figure 8 Figure Supplement 3. 
A
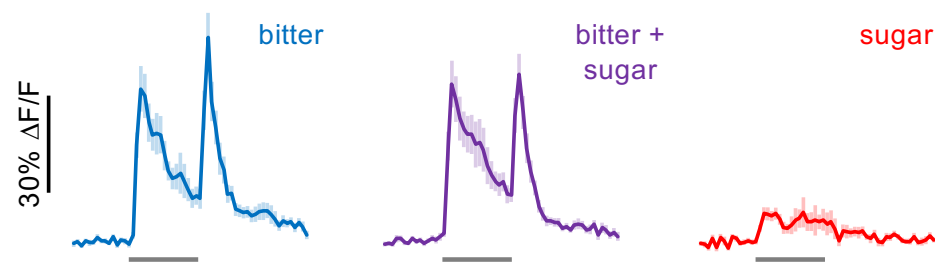

sugar
B

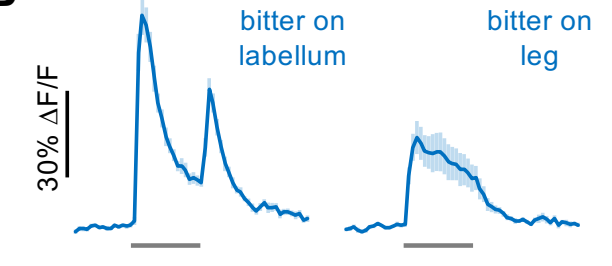

C

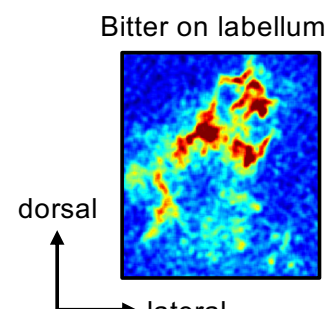

lateral

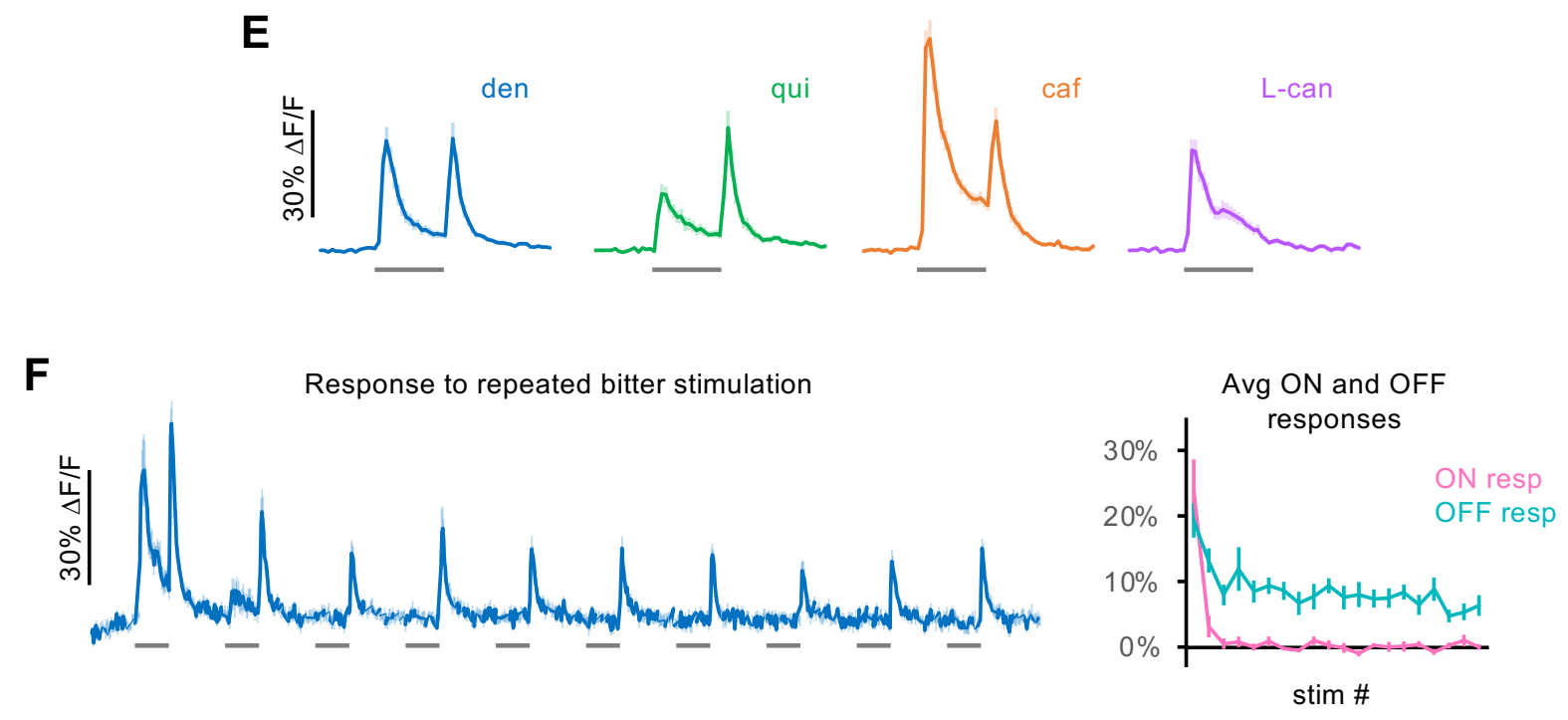

Figure 8 - Figure Supplement 1: Further characterization of mISEZt responses. (A) Responses of mISEZt projections in the SLP when sugar, bitter, or a mixture was applied to the labellum ( $n=16$ trials, 4 flies). (B) Responses of mISEZt projections in the SEZ to labellar versus tarsal bitter stimulation imaged in the same flies ( $n=11-14$ trials, 3 flies). (C) Heatmaps of areas in the SEZ (a single hemisphere was imaged) activated by labellar or tarsal stimulation. Response to $\mathrm{H}_{2} \mathrm{O}$ is shown as the negative control. (D) Heatmaps of areas in the SLP activated by labellar or tarsal stimulation. Pink dotted circles denote individual puncta activated by both organs. (E) Responses of mISEZt neurons to different bitter compounds ( $n=22-23$ trials, 6 flies). (F) Responses of mISEZt neurons to repeated bitter stimulation at $10 \mathrm{sec}$ intervals ( $n=7$ experiments, 5 flies). Left: GCaMP trace. Right: average ON and OFF responses for each stimulus repetition. In panels E-F, tastants were applied to the labellum and SLP projections were imaged. In all panels, R29F12-Gal4 was used to drive UAS-GCaMP6f. 
A

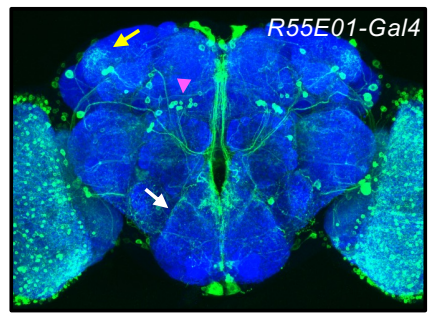

B

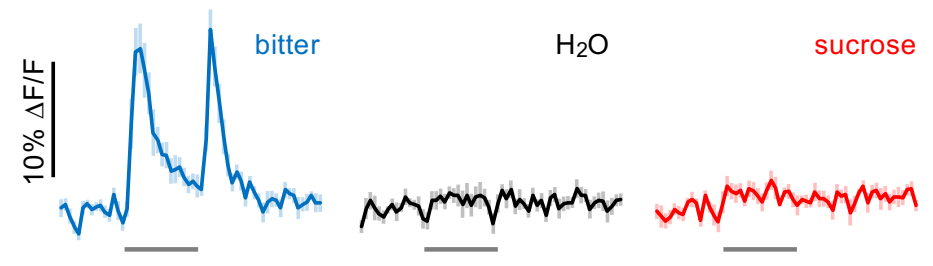

C mISEZt responses using R29F12-R55E01 split-Gal4

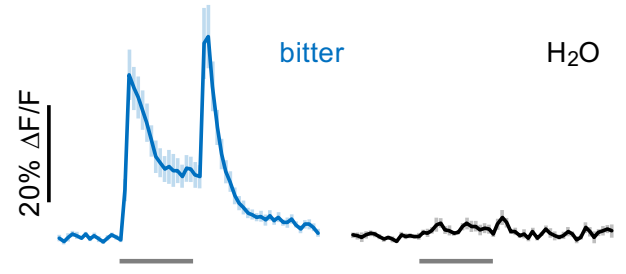

Figure 8 - Figure Supplement 2: Responses of mISEZt cells labeled by R55E01-Gal4 and R29F12-R55E01 split-Gal4. (A) R55E01-Gal4 expression pattern (maximum intensity projection created from images generated by the Janelia FlyLight Project Team). Brain slices at the far anterior and posterior edges are not included in order to maximize visibility of mISEZt cells. Pink arrowhead shows mISEZt cell bodies; white and yellow arrows show their projections in the SEZ and SLP, respectively. (B) Taste responses of mISEZt projections in the SLP using R55E01-Gal4 driving UAS-GCaMP6f (n = 12 trials, 3 flies). (C) Taste responses of mISEZt projections in the SEZ using R29F12-R55E01 split-Gal4 (R29F12-AD; R55E01-DBD) driving UAS-GCaMP6f $(\mathrm{n}=10-15$ trials, 3 flies). In panels $\mathrm{B}-\mathrm{C}$, tastants were applied to the labellum and $10 \mathrm{mM}$ denatonium was used as the bitter stimulus. 
A

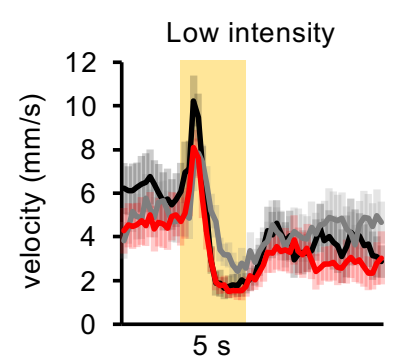

Forward speed

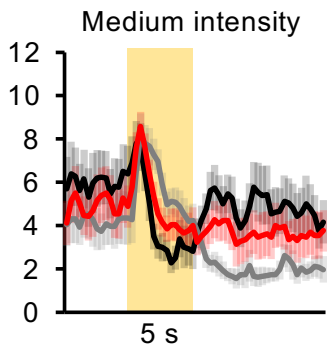

Turning
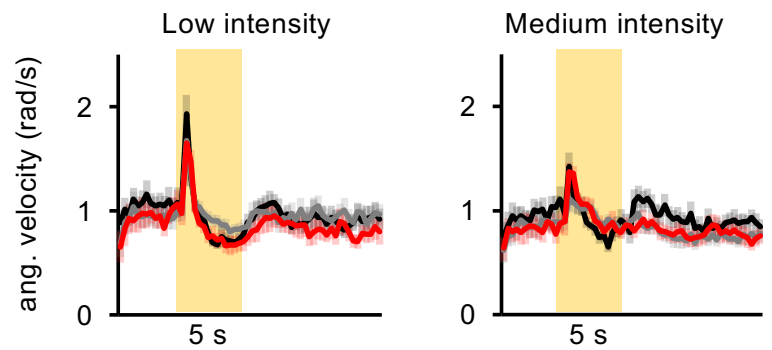

B

Post-light speed

C

Innate preference

Low intensity

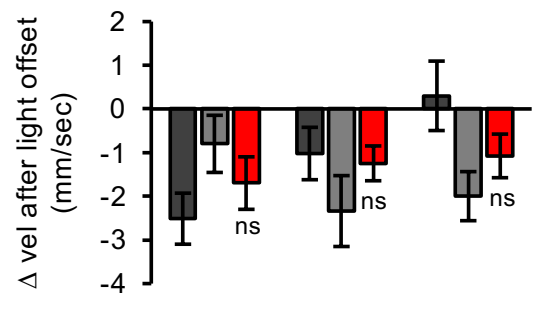

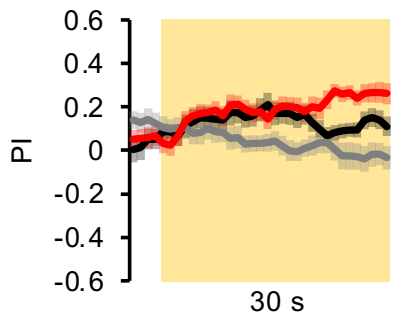

Medium intensity

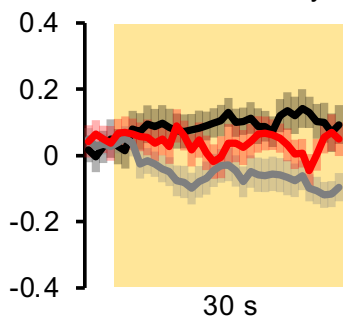

split-Gal4/+ $\quad$ UAS-Chrim/+ split-Gal4/UAS-Chrim

Figure 8 - Figure Supplement 3: Further characterization of behavioral effects induced by mISEZt bitter neuron activation. (A-C) mISEZt bitter neurons were activated using R29F12-R55E01 split-Gal4 driving UAS-Chrimson. (A) Effects of low and medium intensity light stimulation on forward velocity (left) and turning (right; $n=11-12$ trials). Effects of high intensity light are shown in Figure 8G. (B) Change in locomotion at light offset ( $n=11-12$ trials). (C) Innate preference for low and medium intensity light stimulation ( $n=22-24$ trials, $11-12$ sets of flies). Preference for high intensity light is shown in Figure $8 \mathrm{H}$. 